\title{
THE CANONICAL MAP AND HORIKAWA SURFACES IN POSITIVE CHARACTERISTIC
}

\author{
CHRISTIAN LIEDTKE
}

\begin{abstract}
We extend fundamental inequalities related to the canonical map of surfaces of general type to positive characteristic. Next, we classify surfaces on the Noether lines, i.e., even and odd Horikawa surfaces, in positive characteristic. We describe their moduli spaces and the subspaces formed by surfaces whose canonical maps are inseparable. Moreover, we compute their Betti-, deRhamand crystalline cohomology. Finally, we prove lifting to characteristic zero and show that the moduli spaces are topologically flat over the integers.
\end{abstract}

\section{INTRODUCTION}

A detailed classification of surfaces of general type, even over the complex numbers, still looks hopeless. A more modest aim is to prove (in-)equalities holding between their fundamental invariants. Next, one would like to classify at least those surfaces that are extremal with respect to these inequalities. Even over the complex numbers, this is still far from complete and nearly nothing is known over fields of positive characteristic.

One of these fundamental inequalities for minimal surfaces of general type is

$$
\text { Noether's inequality } K_{X}^{2} \geq 2 p_{g}(X)-4
$$

see [Noe] and [BHPV, Chapter VII] for the complex case and [Lie2] for arbitrary characteristic. It generalizes the formula $\operatorname{deg} K_{C}=2 g-2$ for curves.

Depending on the image of the canonical map, there are sharper inequalities by Beauville $[\overline{\mathrm{Bea}}]$ and Castelnuovo $[\overline{\mathrm{C}}]$. As our first result we extend these to arbitrary characteristic

Theorem. Let $X$ be a minimal surface of general type in arbitrary characteristic. In case the canonical image is

$$
\begin{array}{lll}
\text { a curve, } & \text { Beauville's inequality } & K_{X}^{2} \geq 3 p_{g}(X)-6 \\
\text { birational to } X, & \text { Castelnuovo's inequality } & K_{X}^{2} \geq 3 p_{g}(X)-7
\end{array}
$$

holds true.

Not too surprisingly, the main difficulty lies in establishing these inequalities in small characteristics. As in the complex case, the proofs give a structure result for minimal surfaces of general type with $K^{2} \leq 3 p_{g}-7$. We refer to Section 1 for the precise statement.

Date: December 20, 2011.

2000 Mathematics Subject Classification. 14J29, 14 J10. 
Coming back to Noether's inequality, we recall that a minimal surface of general type is called an

$$
\begin{array}{lll}
\text { even Horikawa surface } & \text { if } & K_{X}^{2}=2 p_{g}(X)-4, \quad \text { and } \\
\text { odd Horikawa surface } & \text { if } & K_{X}^{2}=2 p_{g}(X)-3
\end{array}
$$

holds. Both classes together are referred to as surfaces on the Noether lines. Over the complex numbers, they have been roughly classified by Enriques [En] and more thoroughly by Horikawa [Hor1], [Hor2]. A characteristic-free classification of even Horikawa surfaces has already been obtained in [Lie2]. Here, we reprove this result and extend it also to odd Horikawa surfaces.

Theorem. Let $X$ be a surface on the Noether lines in arbitrary characteristic.

(1) If $X$ is an even Horikawa surface then the canonical linear system is base point free.

(2) If $X$ is an odd Horikawa surface with $p_{g} \geq 5$ then the canonical linear system has a unique base point, which can be resolved after one blow-up.

In both cases the canonical map is generically finite of degree 2. Its image is a possibly singular rational surface of degree $p_{g}-2$ in $\mathbb{P}^{p_{g}-1}$.

Using del Pezzo's results on surfaces of degree $n-1$ in $\mathbb{P}^{n}$ it is not difficult to work out the detailed classification, see Section 3. Next, we prove existence:

Theorem. All cases of the previous structure result exist in arbitrary characteristic. Moreover, in characteristic 2 all cases exist with inseparable as well as separable canonical maps.

However, surfaces with inseparable canonical maps exist only in characteristic 2 and even there they are quite rare. More precisely,

Theorem. Surfaces with inseparable canonical maps form a proper closed subset of the moduli space of even Horikawa surfaces with fixed canonical image. Both moduli spaces are irreducible and unirational.

In fact, we determine the codimension in Section 4 The canonical model of a generic even Horikawa surface with inseparable canonical map has usually lots of $A_{1}$-singularities, whereas the canonical model of a generic even Horikawa surface tends to be smooth. We refer to Section 4 for precise and quantitative statements.

Odd Horikawa surfaces with $p_{g} \leq 4$ fall into more classes and we refer to Section 5 for a detailed and characteristic-free classification. For example, we find surfaces with $p_{g}=K^{2}=3$ and inseparable canonical maps in characteristic 3 .

Using our explicit classification we compute the basic invariants

Theorem. Let $X$ be a surface on the Noether lines. Then

(1) it is algebraically simply connected,

(2) its Picard scheme is reduced,

(3) the Frölicher spectral sequence degenerates at E $E_{1}$-level,

(4) its crystalline cohomology is torsion-free, and

(5) the Betti- and deRham-numbers coincide. 
On the other hand, we give examples in arbitrary large characteristics where the slope spectral sequence from Hodge-Witt to crystalline cohomology does not degenerate at $E_{1}$-level.

Finally, we prove lifting:

Theorem. Let $X$ be a surface on the Noether lines over $k=\bar{k}$. Then

(1) its canonical model lifts over the Witt ring $W(k)$, and

(2) X lifts via an algebraic space over a possibly ramified extension of $W(k)$.

As a direct consequence we obtain

Theorem. The moduli spaces of surfaces on the Noether lines are topologically flat over Spec $\mathbb{Z}$.

Unfortunately, in order to prove flatness and to determine the scheme structure of these moduli spaces, we have to understand contributions of singularities to the deformation theory of canonical models better. This will be dealt with in future work.

The article is organized as follows:

In Section 11 we extend Beauville's results circling around Castelnuovo's inequality [Bea, Section 5] to arbitrary characteristic. In particular, we obtain the structure result for surfaces with $K^{2} \leq 3 p_{g}-7$.

In Section 2 we introduce an easy method to construct inseparable morphisms of degree $p$ using generic $\alpha_{\mathcal{L}}$-torsors. We use Cartier's canonical connection to describe their singularities and prove Bertini-type theorems on generic singularities. This section is independent from the rest of the article and will be useful for the construction of surfaces in many other contexts.

From Section 3 on we discuss surfaces on the Noether lines. In Section 3 their basic structure result is proven (assuming $p_{g} \geq 5$ for odd Horikawa surfaces) and existence is shown in arbitrary characteristic. In characteristic 2 we show that all types of surfaces exist with separable as well as inseparable canonical maps.

In Section 4 we give a characteristic-free description of the moduli space of even Horikawa surfaces with fixed canonical image. Moreover, we describe the proper subset formed by surfaces with inseparable canonical map. Finally, we determine the singularities on generic canonical models in these moduli spaces.

In Section 5 we classify the remaining surfaces on the Noether lines, namely odd Horikawa surfaces with $p_{g} \leq 4$ and establish their existence.

In Section 6 we show that the Frölicher spectral sequence for surfaces on the Noether lines degenerates at $E_{1}$. Next, we compute their Betti-numbers, deRhamnumbers and deduce that their crystalline cohomology groups are torsion free. Finally, we give examples in arbitrary large characteristic where the slope spectral sequence does not degenerate at $E_{1}$.

In Section 7 we prove lifting of the canonical models over the Witt ring. This immediately implies that their moduli spaces are topologically flat over Spec $\mathbb{Z}$.

Finally, Appendix A contains a couple of results on double covers, which are somewhat scattered over the literature. Also, we extend results on canonical resolution of Du Val singularities to characteristic 2. 
Acknowledgements. I thank Fabrizio Catanese for a lively discussion at the University of Warwick, which led to the results in Section 4, Also, I thank Torsten Ekedahl and the referee for comments. Finally, I gratefully acknowledge funding from DFG under research grant LI 1906/1-1 and thank the department of mathematics at Stanford university for kind hospitality.

\section{THE CANONICAL MAP}

In this section we establish several classical results on the canonical map of a surface of general type in positive characteristic. Basically, the classical proofs also work in positive characteristic, although a little care is needed since generic fibers of morphisms may no longer be smooth and the vanishing results of Kodaira, Mumford and Ramanujam are not at disposal.

First case: the canonical image is one-dimensional. Our first result is due to Beauville [Bea, Lemme 5.3] over the complex numbers. The main difficulty extending this result to positive characteristic are inseparability possibilities in characteristic 2 , on which we comment during our proof.

Theorem 1.1. Let $X$ be a minimal surface of general type. If the canonical linear system of $X$ defines a rational map with one-dimensional image, then Beauville's inequality

$$
K_{X}^{2} \geq 3 p_{g}-6
$$

holds true.

Proof. Let $\widetilde{X} \rightarrow X$ be the resolution of indeterminacy and $p: \widetilde{X} \rightarrow B$ be the Stein factorization of the canonical map. The generic fiber is a connected and geometrically integral curve $F$. We denote its arithmetic genus by $p_{a}(F)$.

If the canonical map has basepoints, then $B$ is rational. Writing $\left|K_{X}\right|=Z+$ $|a F|$, Riemann-Roch on $B$ yields $a=p_{g}-1$. Moreover, we calculate

$$
K_{X}^{2} \geq a K_{X} F \geq a^{2} F^{2}=\left(a F^{2}\right)\left(p_{g}-1\right)
$$

We are done if $a F^{2} \geq 3$ and the result is trivial for $a=1$. In the remaining case $a=2, F^{2}=1$ we find $K_{X}^{2} \geq 4=3 p_{g}-5$. This establishes the desired inequality.

Thus, we may assume that the canonical linear system defines a morphism. We write it as $\left|K_{X}\right|=Z+|a F|$, where $Z$ denotes the fixed part. Hence there exists a divisor $D_{a}$ of degree $a$ on the base $B$ such that $K_{X} \sim Z+p^{*} D_{a}$. By RiemannRoch on $B$ we obtain $a \geq p_{g}+p_{a}(B)-1$.

In case $p_{a}(F) \geq 3$ we are done since then

$$
K_{X}^{2} \geq a K_{X} F \geq 4 a \geq 4\left(p_{g}-1\right) .
$$

Hence we may assume $p_{a}(F)=2$. We write the canonical system as $\left|K_{X}\right|=$ $V+H+|a F|$, where $V+H$ denotes the fixed part of the canonical system decomposed into a vertical component $V$ and a horizontal component $H$. From $K_{X} F=2$ we obtain $H F=2$. 
In case $H$ is reduced, it is an irreducible curve or the the sum of two irreducible curves. Hence we obtain

$$
\begin{array}{ll}
H K_{X}+H^{2} & \geq-4 \\
H K_{X}-H^{2} & =H V+2 a \geq 2 a
\end{array} \quad \text { by the adjunction formula }
$$

Thus, $H K_{X} \geq a-2$ and we obtain

$$
K_{X}^{2} \geq H K_{X}+2 a \geq 3 a-2 \geq 3 p_{g}-5
$$

It remains to treat the case $H=2 C$, where $C$ is an irreducible curve that defines a section of the fibration.

So far we followed Beauville's proof [Bea, Lemme 5.3]. However, the generic fiber may not be smooth, which can happen only in characteristic $p \leq 5$ by Tate's theorem on genus change in inseparable field extensions and $p_{a}(F)=2$. Moreover, in characteristic 2 it could happen that the map of degree 2 onto a ruled surface that he constructs becomes inseparable.

Instead, we consider $p_{*} \omega_{X}$ on $B$. For every $b \in B$ the fiber $F_{b}=p^{-1}(b)$ has a reduced component (the curve $C$ defines a section), and hence $h^{0}\left(F_{b}, \mathcal{O}_{F_{b}}\right)=1$. Since the Euler characteristic is constant in flat families we obtain $h^{0}\left(F_{b}, \omega_{F_{b}}\right)=2$ for all $b \in B$. The adjunction formula yields

$$
\left.\left.\omega_{X}\right|_{F_{b}} \cong\left(\omega_{X} \otimes \mathcal{O}_{X}\left(F_{b}\right)\right)\right|_{F_{b}} \cong \omega_{F_{b}} .
$$

The global sections being 2-dimensional in every fiber, Grauert's theorem [Hart, Corollary III.12.9] implies that $p_{*} \omega_{X}$ is locally free of rank 2 on $B$ and that for all $b \in B$ the natural map

$$
p_{*} \omega_{X} \otimes_{\mathcal{O}_{B}} k(b) \rightarrow H^{0}\left(F_{b},\left.\omega_{X}\right|_{F_{b}}\right)
$$

is an isomorphism.

Hence the natural morphism $p^{*} p_{*} \omega_{X} \rightarrow \omega_{X}$ defines a rational map $X-\rightarrow$ $S:=\mathbb{P}\left(p_{*} \omega_{X}\right)$, where $S$ is a ruled surface over $B$. On the generic fiber over $B$ it induces the canonical map, which is finite of degree 2 onto $\mathbb{P}_{k(B)}^{1}$ (the generic fiber is a regular curve with $p_{a}=2$ ). Thus, $X \rightarrow S$ is a generically finite, possibly only rational map of degree 2 .

For every $b \in B$ the fiber $F_{b}$ is a Gorenstein curve with $h^{0}\left(\omega_{F_{b}}\right)=2$. Intersecting $C$ with $F_{b}$ we obtain a Cartier divisor $C_{b}$ of degree 1 on $F_{b}$. Furthermore, we have $h^{0}\left(\omega_{F_{b}}\left(-C_{b}\right)\right) \leq 1$, which follows from Riemann-Roch in case $h^{1}$ of this sheaf is zero and from Clifford's inequality in case $h^{1}$ does not vanish. We stress that this is true for every fiber since the theorems of Riemann-Roch and Clifford hold for Gorenstein curves.

For a suitable blow-up $\epsilon: \widehat{X} \rightarrow X$, the rational map extends to a morphism $\pi: \widehat{X} \rightarrow S$. From the previous discussion we see that for every $b \in B$, the Cartier divisor $C_{b}$ on $F_{b}$ is not a base point for $\omega_{F_{b}}$. In particular, we do not have to blow up points on $C$ in the resolution $\epsilon$ of indeterminacy and it follows that the total transform $\epsilon^{*}(C)=\widehat{C}$ is an irreducible curve on $\widehat{X}$.

Now, we follow again [Bea, Lemme 5.3]. From the case-by-case-analysis above we still have

$$
K_{\widehat{X}}=\widehat{V}+2 \widehat{C}+\widehat{p}^{*} D_{a}
$$


where $\widehat{V}$ is a vertical divisor for $\widehat{p}$, and where $\widehat{V}+\widehat{C}$ is the fixed part of $\left|K_{\widehat{X}}\right|$. On the other hand, we know that

$$
\left|K_{\widehat{X}}\right|=\pi^{*}\left|K_{S}+\delta\right|
$$

for some divisor $\delta$ on $S$. We conclude that there exists a curve $\Gamma$ on $S$ that defines a section of $q$ and a divisor $W$ that is vertical for $q$ such that

$$
K_{S}+\delta=W+\Gamma+q^{*} D_{a}, \quad \pi^{*} \Gamma=2 \widehat{C} .
$$

Applying Riemann-Roch to $E:=\Gamma+q^{*} D_{a}$ on $S$ we compute

$$
a+1 \geq h^{0}\left(S, \mathcal{O}_{S}(E)\right) \geq \chi\left(\mathcal{O}_{S}(E)\right)=\chi\left(\mathcal{O}_{S}\right)+\frac{1}{2}\left(E^{2}-E K_{S}\right) .
$$

Denote by $b$ the genus of $B$. We have $\Gamma^{2}+\Gamma K_{S}=2 b-2$, hence $E^{2}-E K_{S}+4 a=$ $2 \Gamma^{2}-2(b-1)+4 a$. From the previous inequality we get $a+1 \geq 1-b+\Gamma^{2}+$ $(1-b)+2 a$ and thus $\Gamma^{2} \leq 2 b-1-a$. Coming back to $X$ we deduce

$$
C^{2}=\widehat{C}^{2} \leq b-\frac{1}{2}(a+1),
$$

whence $C K_{X} \geq b-2+\frac{1}{2}(a+1)$ by the adjunction formula. Finally, we obtain

$$
K_{X}^{2} \geq 2 C K_{X}+2 a \geq 3 a-3 \geq 3 p_{g}-6
$$

and are done.

Remark 1.2. As in [Bea, Remarque 5.4] it follows that if the canonical map of a minimal surface of general type with $\chi\left(\mathcal{O}_{X}\right) \geq 2$ is composed with a pencil over a curve of genus $b \geq 1$ then

$$
K_{X}^{2} \geq 3 p_{g}+5(b-1)
$$

holds true.

Second case: the canonical image is two-dimensional. Let us first slightly extend the notion of hyperelliptic curves. We refer to [Lie2, Section 1] for details.

Definition 1.3. A reduced and irreducible curve $C$, proper over an algebraically closed field, is called hyperelliptic if $p_{a}(C) \geq 2$ and if there exists a morphism of degree 2 from $C$ onto $\mathbb{P}^{1}$.

Following [Bea, Théorème 5.5] and [HorQ, Lemma 2], we now extend several results, which are due to Noether, Castelnuovo, Horikawa, and Beauville over the complex numbers, to arbitrary characteristic.

Theorem 1.4. Let $X$ be a minimal surface of general type and assume that the image of the canonical map is a surface. Let $\pi: \widetilde{X} \rightarrow X$ be a resolution of indeterminacy of the canonical linear system and write

$$
\left|\pi^{*} K_{X}\right|=|L|+F
$$

where $F$ denotes the fixed part. Then Noether's inequality

$$
K_{X}^{2} \geq L^{2} \geq 2 p_{g}-4
$$

holds true. Moreover, 
(1) If $L^{2}=2 p_{g}-4$ then a general member of $|L|$ is a reduced and irreducible Gorenstein curve, which is hyperelliptic.

(2) If $K_{X}^{2}=L^{2}$ then $\left|K_{X}\right|$ is basepoint free.

(3) If $|L|$ defines a birational morphism then Castelnuovo's inequality

$$
K_{X}^{2} \geq L^{2} \geq 3 p_{g}-7
$$

holds true.

Proof. By Bertini's theorem [Jou, Théorème I.6.10], a general member $C$ of $|L|$ is reduced and irreducible. Being a divisor on a smooth surface, $C$ is Gorenstein.

Then we can conclude as in the proof of [HorQ, Lemma 2] that $K^{2} \geq L^{2} \geq$ $2 p_{g}-4$ and that $C$ is hyperelliptic (in the sense of Definition 1.3) if $L^{2}=2 p_{g}-4$ holds, cf. also the proof of [Lie2, Theorem 2.3].

In order to prove assertion (2), we note that

$$
H^{2}\left(\widetilde{X}, \mathcal{O}_{\widetilde{X}}\left(K_{\widetilde{X}}+n L\right)\right)=H^{0}\left(\widetilde{X}, \mathcal{O}_{\widetilde{X}}(-n L)\right)=0 \quad \text { for all } n \geq 1
$$

by Serre duality. By Riemann-Roch we have

$$
\chi\left(\widetilde{X}, \mathcal{O}_{\widetilde{X}}\left(K_{\widetilde{X}}+n L\right)\right)-\chi\left(\widetilde{X}, \mathcal{O}_{\widetilde{X}}\right)=\frac{n}{2} L(E+F)+\frac{n^{2}+n}{2} L^{2},
$$

where $E$ denotes the divisor contained in $\operatorname{exc}(\pi)$ such that $K_{\widetilde{X}}=\pi^{*} K_{X}+E$ holds true.

By [Ek2, Proposition I.1.7] we have $h^{1}\left(-n K_{X}\right)=0$ for $n \geq 2$ and then Riemann-Roch together with (1) yield

$$
\begin{aligned}
\frac{n(n+1)}{2} K_{X}^{2} & =h^{0}\left(X, \mathcal{O}_{X}\left((n+1) K_{X}\right)\right)-\chi\left(\mathcal{O}_{X}\right) \\
& \geq h^{0}\left(\widetilde{X}, \mathcal{O}_{X}\left(K_{\tilde{X}}+n L\right)\right)-\chi\left(\mathcal{O}_{\tilde{X}}\right) \\
& \geq \chi\left(\widetilde{X}, \mathcal{O}_{X}\left(K_{\tilde{X}}+n L\right)\right)-\chi\left(\mathcal{O}_{\tilde{X}}\right) .
\end{aligned}
$$

Combining with (2) we obtain

$$
K_{X}^{2} \geq \frac{1}{n+1} L(E+F)+L^{2}
$$

for $n \geq 2$ and even for $n=1$ except possibly for some surfaces in characteristic 2 [Ek2, Theorem II.1.7]. Now, if $L^{2}=K^{2}$, we have $L E=L F=0$. First, $L E=0$ implies that $L$ can be considered as a divisor on $X$. Hence $\pi$ is the identity. Second, $|K|=|L|+F$ with $L F=0$ and the connectedness of canonical divisors implies $F=0$. This proves that $|K|$ has no base point in case $L^{2}=K^{2}$ holds.

To prove (3), we note that the proof of [Bea, Théorème 5.5] works in arbitrary characteristic.

Castelnuovo's inequality and Beauville's results. The previous results extend [Bea, Théorème 5.5] and [Bea, Remarque 5.6] to arbitrary characteristic:

Theorem 1.5. Let $X$ be a minimal surface of general type.

(1) If $K^{2}=3 p_{g}-7$ then the canonical map is either a rational map of degree 2 onto a ruled surface or a birational morphism. 
(2) If $K^{2}<3 p_{g}-7$ then the canonical map is a rational map of degree 2 onto a ruled surface.

Over the complex numbers, surfaces with $K^{2}=3 p_{g}-7$ and birational canonical map have been studied by Castelnuovo $[\bar{C}]$. They have been classified without the birationality assumption by Ashikaga, Horikawa, Konno and others, see [AK] for details and further references. It seems plausible that their classification in positive characteristic is the same as over the complex numbers. We shall deal with the case $K^{2}=3 p_{g}-7=5$ in Section 5 below.

\section{THE GEOMETRY OF $\alpha_{\mathcal{L}}$-TORSORS}

In this section we describe a handy and useful method to construct inseparable covers using $\alpha_{\mathcal{L}}$-torsors. These are inseparable versions of cyclic Galois covers. Even if the base of the cover is smooth and $\mathcal{L}$ is sufficiently ample, these covers are almost always singular. However, the singularities are controlled by Cartier's canonical connection. Moreover, in the surface case we will see that in nice and generic situations we may assume these singularities to be Du Val.

In characteristic zero, cyclic Galois covers are omnipresent for the construction and classification of algebraic varieties. In positive characteristic $p$, inseparable covers are equally important and the simplest type are morphisms that are locally torsors under $\mu_{p}$ or $\alpha_{p}$. Now, the group action of a Galois morphism between normal varieties is completely determined at their function fields. This is in contrast to inseparable morphisms: a morphism that is Zariski-locally an $\alpha_{p}$-torsor may not give rise to a global $\alpha_{p}$-action. This leads to the notion of $\alpha_{\mathcal{L}}$-torsors introduced in [Ek2, Section 0], which we recall here for the reader's convenience:

Let $k$ be an algebraically closed field of positive characteristic $p$ and let $S$ be a smooth variety over $k$ of arbitrary dimension. As usual, we denote by $F: S \rightarrow$ $S^{(p)}$ the $k$-linear Frobenius morphism. Since $k$ is perfect we shall identify $S$ and $S^{(p)}$ whenever needed.

Now, given an invertible sheaf $\mathcal{L}$ on $S$ we consider $\mathcal{L}$ and $\mathcal{L}^{\otimes p}$ as relative group schemes over $S$. Then Frobenius induces a short exact sequence (in the flat topology) of group schemes over $S$

$$
0 \rightarrow \alpha_{\mathcal{L}} \rightarrow \mathcal{L} \rightarrow \mathcal{L}^{\otimes p} \rightarrow 0 .
$$

This $\alpha_{\mathcal{L}}$ is a finite flat and infinitesimal group scheme of relative length $p$ over $S$, cf. [Ek2, Section 0.(1.5)]. Locally in the Zariski-toplogy this group scheme is isomorphic to $\alpha_{p}$, whence the name.

Remark 2.1. In characteristic 2, every finite inseparable morphism $X \rightarrow S$ of degree 2, where $S$ is smooth and $X$ is Cohen-Macaulay is an $\alpha_{\mathcal{L}}$-torsor for a suitable invertible sheaf $\mathcal{L}$ by [Ek2, Proposition 0.1.11]. This is analogous to the fact that every such morphism of degree 2 in characteristic $\neq 2$ is Galois.

Torsors under $\alpha_{\mathcal{L}}$ are classified by cohomology classes in $H_{\mathrm{fl}}^{1}\left(S, \alpha_{\mathcal{L}}\right)$, where the subscript $f$ refers to the fact that flat topology is required to define this cohomology 
group (in order to trivialize such torsors). Taking cohomology in (3) we obtain

$$
\ldots \rightarrow H^{0}\left(S, \mathcal{L}^{\otimes p}\right) \stackrel{\delta}{\rightarrow} H_{\mathrm{fl}}^{1}\left(S, \alpha_{\mathcal{L}}\right) \rightarrow H^{1}(S, \mathcal{L}) \rightarrow \ldots
$$

Given an open affine set $U \subseteq S$ we have $H^{1}\left(U,\left.\mathcal{L}\right|_{U}\right)=0$ and thus the $\alpha_{\mathcal{L}}$-torsor restricted to $U$ arises as $\delta(s)$ for a section $s \in H^{0}\left(U, \mathcal{L}^{\otimes p}\right)$. Choosing a local generator $t$ of $\mathcal{L}$ (after possibly passing to an open affine cover of $U$ ), the torsor over $U$ is given by

$$
\mathcal{O}_{U}[t] /\left(t^{p}-f\right) \rightarrow \mathcal{O}_{U}
$$

for some $f \in \mathcal{O}_{U}$. The elements $d\left(s / t^{p}\right) \in \Omega_{U}^{1}$ glue to a well-defined global $\mathcal{O}_{S}$-morphism

$$
\mathcal{L}^{\otimes(-p)} \rightarrow \Omega_{S / k}^{1}
$$

whose zeroes correspond to the singularities of $S$. The annihilator of this morphism is a subsheaf inside the tangent sheaf $\Theta_{S / k}$, which is the $p$-closed foliation associated to the inseparable morphism $\pi$, cf. the discussion after [Ek2, Lemma I.2.5]. Viewing this assignment of a (local) section $s$ of $\mathcal{L}^{\otimes p}$ to a (local) morphism (6) as a function of $s$, i.e., varying the $\alpha_{\mathcal{L}}$-torsor we obtain the following

Proposition 2.2. The map

$$
\nabla: \mathcal{L}^{\otimes p} \rightarrow \mathcal{H} \operatorname{om}\left(\mathcal{L}^{\otimes(-p)}, \Omega_{S / k}^{1}\right) \cong \Omega_{S / k}^{1} \otimes \mathcal{L}^{\otimes p}
$$

is an integrable connection of p-curvature zero. Considering $\mathcal{L}$ as invertible sheaf on $X^{(p)}$, we may identify $\mathcal{L}^{\otimes p}$ on $X$ with $F^{*} \mathcal{L}$. Then horizontal sections of $\nabla$ form an invertible sheaf on $X^{(p)}$ and we obtain

$$
\left(F^{*} \mathcal{L}\right)^{\nabla} \cong \mathcal{L}
$$

Thus, $\nabla$ can be identified with the Cartier connection on $F^{*} \mathcal{L}$.

Proof. For a local parameter $t \in \mathcal{L}$ and a section $s=f \cdot t^{p}$ of $\mathcal{L}^{\otimes p}$ with $f \in \mathcal{O}_{S}$ we have $\nabla(s)=d f \otimes t^{p}$. A straight forward calculation shows that $\nabla$ is an integrable connection of $p$-curvature zero. Moreover, its horizontal sections are of the form $f^{p} \cdot t^{p}$, i.e., come from $\mathcal{L}$, considered as an invertible sheaf on $X^{(p)}$. These properties characterize $\nabla$ as Cartier's connection [Ka, Theorem 5.1].

Let us recall that a cyclic Galois covering $\pi: X \rightarrow S$ of order $\ell$ prime to char $(k)$ is also given by an invertible sheaf $\mathcal{L}$ and that $\pi_{*} \mathcal{O}_{X}$ as a $\mathcal{O}_{S}$-module is isomorphic to the direct sum of $\mathcal{L}^{-i}$ where $i$ runs from 0 to $\ell-1$. This direct sum decomposition comes from the fact that the Galois action decomposes $\pi_{*} \mathcal{O}_{S}$ into eigensheaves, all of which turn out to be invertible if $S$ is smooth.

On the other hand, every $\alpha_{\mathcal{L}}$-torsor defines an extension class

$$
\left[0 \rightarrow \mathcal{O}_{S} \rightarrow \mathcal{E} \rightarrow \mathcal{L}^{\vee} \rightarrow 0\right] \in \operatorname{Ext}^{1}\left(\mathcal{L}^{\vee}, \mathcal{O}_{S}\right)
$$

which splits after Frobenius pullback [Ek2, Section 0.(1.6)]. We reinterpret (4) as

$$
\ldots \rightarrow H^{0}\left(S, F^{*} \mathcal{L}\right) \stackrel{\delta}{\rightarrow} H_{\mathrm{fl}}^{1}\left(S, \alpha_{\mathcal{L}}\right) \stackrel{e}{\rightarrow} \operatorname{Ext}^{1}\left(\mathcal{L}^{\vee}, \mathcal{O}_{S}\right) \rightarrow \operatorname{Ext}^{1}\left(\mathcal{L}^{\otimes(-p)}, \mathcal{O}_{S}\right) \rightarrow \ldots
$$


Thus, the image of $\delta$ consists of those $\alpha_{\mathcal{L}}$-torsors, whose associated extension class in $\operatorname{Ext}^{1}\left(\mathcal{L}^{\vee}, \mathcal{O}_{S}\right)$ is trivial, i.e., torsors of the form

$$
X \cong \operatorname{Spec} \bigoplus_{i=0}^{p-1} \mathcal{L}^{\otimes(-i)} \rightarrow S
$$

and where the multiplication map on the $\mathcal{O}_{S}$-algebra $\mathcal{O}_{X}$ is globally given by a section $s \in H^{0}\left(S, \mathcal{L}^{\otimes p}\right)$. Here is an important example where the extension class

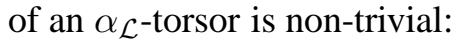

Example 2.3. Let $E / k$ be an elliptic curve. Then $F: E \rightarrow E^{(p)}$ is an $\alpha_{\mathcal{L}}$-torsor for $\mathcal{L}=\mathcal{O}_{E^{(p)}}$ corresponding to a class $\eta \in H_{\mathrm{fl}}^{1}\left(E^{(p)}, \alpha_{\mathcal{L}}\right)$. It satisfies

$$
0 \neq e(\eta) \in \operatorname{Ext}^{1}\left(\mathcal{O}_{E^{(p)}}, \mathcal{O}_{E^{(p)}}\right) \cong H^{1}\left(E^{(p)}, \mathcal{O}_{E^{(p)}}\right),
$$

for otherwise the torsor would be trivial. Depending on whether $E$ is ordinary or supersingular, $F$ can also be interpreted as $\mu_{p^{-}}$or $\alpha_{p}$-torsor, respectively.

Let us now study $\alpha_{\mathcal{L}}$-torsors of the form $\delta H^{0}\left(S, \mathcal{L}^{\otimes p}\right)$, still assuming $S$ to be smooth. Thus, let $s$ be a global section of $\mathcal{L}^{\otimes p}$. Then $\delta(s)$ gives rise to an $\alpha_{\mathcal{L}^{-}}$ torsor $\pi: X \rightarrow S$. We have seen above that the singularities of $X$ correspond to the zeroes of $\nabla(s)$ where $\nabla$ is the Cartier connection on $\mathcal{L}^{\otimes p}$.

From the local description (5) it follows that $X$ is Cohen-Macaulay since $S$ is smooth. As explained in [Ek2, Section 0.(1.6)], every non-trivial $\alpha_{\mathcal{L}}$-torsor over a smooth base is automatically reduced. Clearly, a torsor of the form $\delta(s)$ is nontrivial if and only if $s$ is not equal to $w^{p}$ for some $w \in H^{0}(S, \mathcal{L})$.

If $\nabla(s)$ does not vanish along a divisor on $S$ then the associated $\alpha_{\mathcal{L}}$-torsor $\delta(s)$ is regular in codimension one and thus normal by Serre's criterion.

Note that $\pi: X \rightarrow S$ is everywhere ramified. Thus, despite the description (5), the zero divisor of $s$ does not have a geometric interpretation as branch divisor. On the other hand, let $D \subset S$ be a prime divisor contained in the zero divisor of $s$. If $X$ is normal and $E$ denotes the reduced inverse image of $D$ then $\pi_{*} E=D$ and $\pi^{*} D=p E$.

Let us finally assume that $S$ is a smooth projective surface and $X \rightarrow S$ is an

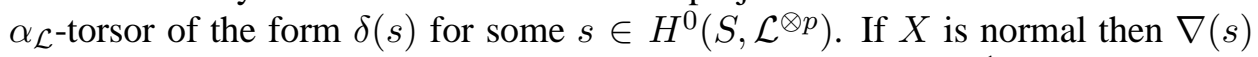
has only isolated zeroes. More precisely, $\nabla(s)$ will have $c_{2}\left(\Omega_{S}^{1} \otimes \mathcal{L}^{\otimes p}\right)$ zeroes, counted with multiplicity. Thus, in general $X$ will not be smooth, even if $\mathcal{L}^{\otimes p}$ is sufficiently ample. On the other hand, the occurring singularities are not too bad as the following Bertini-type result shows.

Theorem 2.4. Let $S$ be a smooth projective surface over an algebraically closed field of characteristic $p>0$. Let $\mathcal{L}$ be an invertible sheaf on $S$ such that

(1) $\forall x \in S$ there exist two sections $s_{1}, s_{2} \in H^{0}\left(S, \mathcal{L}^{\otimes p}\right)$ meeting transversally in $x$

(2) $\forall x \in S$ there exists a section $s \in H^{0}\left(S, \mathcal{L}^{\otimes p}\right)$ with strict normal crossings in $x$. 
Then there exists an open and dense subset $U \subseteq H^{0}\left(S, \mathcal{L}^{\otimes p}\right)$ such that for all $s \in U$ the associated $\alpha_{\mathcal{L}}$-torsor $\delta(s)$ is normal with precisely $c_{2}\left(\Omega_{S}^{1} \otimes \mathcal{L}^{\otimes p}\right) D u$ Val singularities of type $A_{p-1}$ and no further singularities.

PROOF. The first part is similar to the proof [Hart, Theorem II.8.18] of Bertini's theorem: For a closed point $P \in S$ we choose an open affine neighborhood $P \in U$ and (after possibly shrinking $U$ ) a section $f_{0} \in H^{0}(U, \mathcal{L})$ with $f_{0}(P) \neq 0$. Then we define

$$
\begin{array}{ccc}
\varphi_{P}: H^{0}\left(S, \mathcal{L}^{\otimes p}\right) & \rightarrow \mathcal{O}_{S, P} / \mathfrak{m}_{P}^{3} \\
s & \mapsto & \frac{s}{f_{0}^{p}}
\end{array}
$$

viewing the right hand side as 6 -dimensional $k$-vector space. Using the natural embedding of $k$ into $\mathcal{O}_{S, P}$ we form the quotient map $\bar{\varphi}_{P}=\varphi_{P} \bmod k$ to the 5-dimensional vector space $\mathcal{O}_{S, P} /\left(k \oplus \mathfrak{m}_{P}^{3}\right)$.

After choosing generators $x, y \in \mathfrak{m}_{P}$ we may form the Taylor expansion with respect to $x, y$, which, by our particular choice of local parameter $f_{0}^{p}$ of $\mathcal{L}^{\otimes p}$, yields Cartier's connection

$$
\begin{aligned}
\nabla\left(\varphi_{P}(s)\right) & =\left(\frac{\partial \widetilde{s}}{\partial x}(P) \cdot x+\frac{\partial^{2} \widetilde{s}}{\partial x^{2}}(P) \cdot x^{2}+\frac{\partial^{2} \widetilde{s}}{\partial x \partial y}(P) \cdot x y+\ldots\right) \cdot d x \\
& +\left(\frac{\partial \widetilde{s}}{\partial y}(P) \cdot y+\frac{\partial^{2} \widetilde{s}}{\partial y^{2}}(P) \cdot y^{2}+\frac{\partial^{2} \widetilde{s}}{\partial x \partial y}(P) \cdot x y+\ldots\right) \cdot d y
\end{aligned}
$$

Using the assumptions on the existence of sections of $H^{0}\left(S, \mathcal{L}^{\otimes p}\right)$ it is easy to see that the image of $\bar{\varphi}_{P}$ is at least 3-dimensional. Thus, $\operatorname{ker}\left(\bar{\varphi}_{P}\right)$ is of codimension at least 3 in $H^{0}\left(S, \mathcal{L}^{\otimes p}\right)$.

On the other hand, a section $s \in \operatorname{ker}\left(\bar{\varphi}_{P}\right)$ has the property that $\nabla(s)$ has a zero of multiplicity at least two in $P$. Moreover, if the zeroes of $\nabla(s)$ are not isolated around $P$ then also $s \in \operatorname{ker}\left(\bar{\varphi}_{P}\right)$ holds true.

Considering

$$
B:=\left\{(P, s) \mid s \in \operatorname{ker}\left(\bar{\varphi}_{P}\right)\right\} \subseteq S \times H^{0}\left(S, \mathcal{L}^{\otimes p}\right)
$$

with its two projections $\operatorname{pr}_{1}, \operatorname{pr}_{2}$ to $S$ and $H^{0}\left(S, \mathcal{L}^{\otimes p}\right)$, a dimension count shows that $\operatorname{pr}_{2}(B)$ is a proper subset of $H^{0}\left(S, \mathcal{L}^{\otimes p}\right)$. Since we assumed $k$ to be algebraically closed, there exist sections $s \in H^{0}\left(S, \mathcal{L}^{\otimes p}\right)$ where the zeroes of $\nabla(s)$ are isolated of multiplicity one. Since both properties are open it follows in fact that there exists an open and dense subset $U \subseteq H^{0}\left(S, \mathcal{L}^{\otimes p}\right)$ of such sections.

Finally, locally analytically around a zero of $\nabla(s)$ the torsor is given by $z^{p}-x y$, which defines a Du Val singularity of type $A_{p-1}$.

Remarks 2.5. We note that

(1) The conditions on $\mathcal{L}^{\otimes p}$ are fulfilled whenever $\mathcal{L}$ is very ample.

(2) If $V \subseteq H^{0}\left(S, \mathcal{L}^{\otimes p}\right)$ fulfills the assumptions then the conclusion also holds for a generic section of $V$.

(3) For more on generic $\alpha_{\mathcal{L}}$-torsors, we refer to [Ek1, Chapter VI]. Since I was unaware of this reference until pointed out to me by Torsten Ekedahl, there are slight overlaps with my exposition. 
In [Ek2, I.(1.10)] the notion of $\alpha_{\mathcal{L}}$-torsor is generalized to $\alpha_{s}$-torsors, which coincides with the notion of Artin-Schreier covering of simple type in [Ta]. For a global section $s \in H^{0}\left(S, \mathcal{L}^{\otimes(p-1)}\right)$ we define $\alpha_{s}$ to be the relative group scheme over $S$, which is the kernel of

$$
0 \rightarrow \alpha_{s} \rightarrow \mathcal{L} \stackrel{F-s}{\rightarrow} \mathcal{L}^{\otimes p} \rightarrow 0 .
$$

For $s=0$ we obtain $\alpha_{\mathcal{L}}$ but if $s \neq 0$ we obtain a group scheme, which is étale over $S$ outside the zero divisor of $s$. As before, these torsors are classified by cohomology classes $H_{\mathrm{fl}}^{1}\left(S, \alpha_{s}\right)$ and to every $\alpha_{s}$-torsor one obtains an extension class in $\operatorname{Ext}^{1}\left(\mathcal{L}^{\vee}, \mathcal{O}_{S}\right)$. Whenever this extension class is trivial the torsor is globally given as

$$
\text { Spec } \mathcal{O}_{S}[z] /\left(z^{p}-s z+t\right) \rightarrow S
$$

for some global section $t \in H^{0}\left(S, \mathcal{L}^{\otimes p}\right)$. Note that this morphism is branched over the zero divisor of $s$. However, note that if this divisor is smooth this does not necessarily imply that the torsor itself is smooth. On the other hand, only singularities of type $A_{p-1}$ can occur if the zero divisor of $s$ is smooth [Ta, Theorem 2.1]. Let us strengthen this result slightly, whose proof is analogously to that of Theorem 2.4, which is why we leave the proof to the reader.

Theorem 2.6. Let $S$ be a smooth projective surface over an algebraically closed field of characteristic $p>0$. Let $\mathcal{L}$ be an invertible sheaf on $S$ such that

(1) the sheaf $\mathcal{L}$ is generated by global sections,

(2) $\forall x \in S$ there exist two sections $s_{1}, s_{2} \in H^{0}\left(S, \mathcal{L}^{\otimes(p-1)}\right)$ meeting transversally in $x$

Then there exists an open and dense subset $U \subseteq H^{0}\left(S, \mathcal{L}^{\otimes(p-1)}\right) \oplus H^{0}\left(S, \mathcal{L}^{\otimes p}\right)$ such that for all $(s, t) \in U$ the associated split $\alpha_{s}$-torsor is smooth.

Remark 2.7. The conditions are fulfilled whenever $\mathcal{L}$ is very ample.

\section{SuRfaCES ON THE NOETHER Lines}

From now on we study surfaces on the Noether lines. In this section we prove their basic structure result except for some cases with small $p_{g}$, with which we deal in Section 5 . The surfaces of this section are double covers of rational surfaces via their canonical map. Next, we establish existence. Moreover, in characteristic 2 we prove that all classes exist with separable as well as inseparable canonical maps. Finally, we show that they are algebraically simply connected and have reduced Picard schemes.

Let us first recall from [Lie2, Theorem 2.1] that minimal surfaces of general type fulfill Noether's inequality

$$
K_{X}^{2} \geq 2 p_{g}-4
$$

Over the complex numbers, surfaces with $K^{2}=2 p_{g}-4$ have been described by Enriques in [En. Capitolo VIII.11] and a detailed analysis of surfaces with $K^{2} \leq 2 p_{g}-3$ has been carried out by Horikawa in [Hor1] and [Hor2]. In positive characteristic, the description of surfaces with $K^{2}=2 p_{g}-4$ is the same as in characterstic zero [Lie2]. 
Definition 3.1. A minimal surface of general type is called

(1) an even Horikawa surface if $K^{2}=2 p_{g}-4$, and

(2) an odd Horikawa surface if $K^{2}=2 p_{g}-3$.

A surface belonging to one of these classes is said to lie on the Noether lines.

We start with a preliminary analysis of their canonical maps, which is the key to their classification.

Proposition 3.2. Let $X$ be a surface on the Noether lines.

(1) If $X$ is an even Horikawa surface then $p_{g} \geq 3$ and the canonical linear system is basepoint free.

(2) If $X$ is an odd Horikawa surface with $p_{g} \geq 5$ then the canonical linear system has a unique base point, whose indeterminacy can be resolved after one blow-up.

In all these cases the canonical map is generically finite of degree 2 onto a surface of degree $p_{g}-2$ in $\mathbb{P}^{p_{g}-1}$.

Proof. If $X$ is an even Horikawa surface then the canonical map is basepoint free and a general member of the canonical system is a (possibly singular) hyperelliptic curve by Theorem 1.4 The canonical image is a surface which follows from Theorem 1.1 and $p_{g} \geq 3$. Also, the canonical map cannot be birational since the general canonical divisor is hyperelliptic and so $\omega_{X}$ does not restrict to a very ample linear system on it. Furthermore we know

$$
K_{X}^{2}=L^{2} \geq \operatorname{deg} \phi_{1} \cdot \operatorname{deg} \phi_{1}(X) .
$$

But $\phi_{1}(X)$ is a surface inside $\mathbb{P}^{p_{g}-1}$ and thus has degree at least $p_{g}-2$. This leaves only the possibility $\operatorname{deg} \phi_{1}=2$ and $\operatorname{deg} \phi_{1}(X)=p_{g}-2$.

Now, let $X$ be an odd Horikawa surface with $p_{g} \geq 5$. Again, by Theorem 1.1 and Theorem 1.4 the image of the canonical map is a surface and the canonical map cannot be birational. The inequality

$$
2 p_{g}-3=K_{X}^{2} \geq L^{2}=\operatorname{deg} \phi_{1} \cdot \operatorname{deg} \phi_{1}(X) \geq 2 \cdot\left(p_{g}-2\right)
$$

has the only solution $K_{X}^{2}=L^{2}-1, \operatorname{deg} \phi_{1}=2$ and $\operatorname{deg} \phi_{1}(X)=p_{g}-2$. In particular, the canonical system has a unique basepoint.

Remark 3.3. A reduced and irreducible surface in $\mathbb{P}^{n}$ that spans its ambient space has degree at least $n-1$. Surfaces of this minimal degree have been classified by del Pezzo. For $n \geq 3$, the homogeneous ideal defining such a surface of degree $n-$ 1 in $\mathbb{P}^{n}$ is generated by $\frac{1}{2}(n-1)(n-2)$ quadratic polynomials. These polynomials correspond to the $2 \times 2$ minors of a certain $(n-1) \times 2$ matrix for Hirzebruch surfaces and cones over rational normal curves, and the $2 \times 2$ minors of a certain symmetric $3 \times 3$ matrix for the Veronese surface in $\mathbb{P}^{5}$, namely

$$
\left(\begin{array}{llllll}
x_{0,0} & \ldots & x_{0, a_{0}-1} & x_{1,0} & \ldots & x_{1, a_{1}-1} \\
x_{0,1} & \ldots & x_{0, a_{0}} & x_{1,1} & \ldots & x_{1, a_{1}}
\end{array}\right) \quad \text { and } \quad\left(\begin{array}{lll}
x_{0} & x_{1} & x_{2} \\
x_{1} & x_{3} & x_{4} \\
x_{2} & x_{4} & x_{5}
\end{array}\right) .
$$

We refer to $[\overline{\mathrm{EH}}]$ for details and further references. 
Now, it is not so difficult to show that the first Betti number of a surface on the Noether lines is zero. Although this implies that the Picard scheme is zerodimensional we could still have the possibility of a non-trivial $H^{1}\left(X, \mathcal{O}_{X}\right)$ caused by a non-reduced Picard scheme. We start with an argument from the proof of [Bo, Theorem 14].

Lemma 3.4. Let $X$ be a surface on the Noether lines with $h^{1}\left(\mathcal{O}_{X}\right)=0$. Then $X$ is algebraically simply connected, i.e., its étale fundamental group is trivial.

Proof. Let $X$ be a surface with $h^{1}\left(\mathcal{O}_{X}\right)=0$ and $\widehat{X} \rightarrow X$ be an étale cover of degree $m$. Then we compute $\chi\left(\mathcal{O}_{\widehat{X}}\right)=m \chi\left(\mathcal{O}_{X}\right)$ and $K_{\widehat{X}}^{2}=m K_{X}^{2}$. Using Noether's inequality we obtain

$m\left(1+p_{g}(X)\right)=1-h^{1}\left(\mathcal{O}_{\widehat{X}}\right)+p_{g}(\widehat{X}) \leq 1+p_{g}(\widehat{X}) \leq \frac{1}{2} K_{\widehat{X}}^{2}+3=\frac{m}{2} K_{X}^{2}+3$.

If $X$ is a surface on the Noether lines then this inequality holds for $m=1$ only. Thus, every étale cover of $X$ is trivial and hence $X$ is algebraically simply connected.

Proposition 3.5. Let $X$ be a surface on the Noether lines, which is

(1) an even Horikawa surface or

(2) an odd Horikawa surface with $p_{g} \geq 5$.

Then $b_{1}(X)=h^{1}\left(\mathcal{O}_{X}\right)=0$ and the Picard scheme of $X$ is reduced. Moreover, $X$ is algebraically simply connected.

PROOF. An even Horikawa surface fulfills $p_{g} \geq 3$, i.e., we may assume $p_{g} \geq 3$ in any case. Then the image of the canonical map is a surface by Theorem 1.1 in case $p_{g} \geq 4$ or in general by Noether's inequality [Lie2, Theorem 2.1].

From Proposition 3.2 we know that the canonical image is a surface of degree $p_{g}-2$ inside $\mathbb{P}^{p_{g}-1}$. By Remark 3.3 we can find a basis of the $p_{g}$-dimensional vector space $H^{0}\left(X, \omega_{X}\right)$ such that the quadratic relations among them are precisely the ones given by $2 \times 2$ minors described there. Using the multiplication map $\left|K_{X}\right| \times\left|K_{X}\right| \rightarrow\left|2 K_{X}\right|$ and the quadratic relations we get

$$
p_{2}(X):=h^{0}\left(X, \omega_{X}^{\otimes 2}\right) \geq 3 p_{g}-3 .
$$

Together with Riemann-Roch we find

$$
K_{X}^{2}+\chi\left(\mathcal{O}_{X}\right)+h^{1}\left(X, \omega_{X}^{\vee}\right)=p_{2} \geq 3 p_{g}-3 .
$$

We have $h^{1}\left(\omega_{X}^{\vee}\right) \leq 1$ by [Ek2, Theorem II.1.7] and this dimension is zero except for a certain class of surfaces with $\chi\left(\mathcal{O}_{X}\right) \leq 1$ in characteristic 2. However, (7) gives us already $h^{1}\left(\mathcal{O}_{X}\right) \leq 2$ for our surfaces. In particular, they fulfill $\chi\left(\mathcal{O}_{X}\right) \geq 2$ and hence $h^{1}\left(\omega_{X}^{\vee}\right)=0$. Applying (7) once more we obtain $h^{1}\left(\mathcal{O}_{X}\right)=0$ for even Horikawa surfaces and $h^{1}\left(\mathcal{O}_{X}\right) \leq 1$ for odd Horikawa surfaces with $p_{g} \geq 5$.

We have to exclude the possibility of odd Horikawa surfaces with $p_{g} \geq 5$ and $h^{1}\left(\mathcal{O}_{X}\right)=1$. In this case we would have $p_{2}=3 p_{g}-3$, implying that the bicanonical map of $X$ is the composition of the canonical map followed by the second Veronese map. By Proposition 3.2, the canonical map of such a surface has a 
basepoint and hence so has the bicanonical map. However, this contradicts [S-B] Theorem 26].

Hence $h^{1}\left(\mathcal{O}_{X}\right)=0$ holds true in all cases, and the surfaces are algebraically simply connected by Lemma 3.4

Remarks 3.6. Let us note that

(1) Over the complex numbers, these surfaces are even topologically simply connected by [Hor1, Theorem 3.4] and [Hor2, Theorem 4.8].

(2) There do exist minimal surfaces of general type with $K^{2}=h^{01}=p_{g}=1$ and $b_{1}=0$ in characteristic 5, namely non-classical Godeaux surfaces, see [Mi] and [Lie3]. Thus, not "too far away" from the Noether lines nonreduced Picard schemes show up.

From del Pezzo's analysis of surfaces of minimal degree and Proposition 3.2 we get an explicit description of our surfaces.

By Proposition 3.2, double covers play a central role. We refer to Appendix A for a characteristic-free discussion. In particular, the notion of an invertible sheaf associated with a double cover is introduced there. We remind the reader that the notion of branch divisor is not well-defined for inseparable morphisms.

For an integer $d \geq 0$, we let $\mathbb{F}_{d}$ be the Hirzebruch surface $\mathbb{P}_{\mathbb{P}^{1}}\left(\mathcal{O}_{\mathbb{P}^{1}} \oplus \mathcal{O}_{\mathbb{P}^{1}}(d)\right)$. This $\mathbb{P}^{1}$-bundle over $\mathbb{P}^{1}$ has a section $\Delta_{0}$ with self-intersection $\Delta_{0}^{2}=-d$, which is unique if $d$ is positive. We denote by $\Gamma$ the class of a fiber of this $\mathbb{P}^{1}$-bundle.

Theorem 3.7. Let $X$ be an even Horikawa surface. Then the canonical linear system is a morphism and its canonical image $S=\phi_{1}(X)$ is a surface of degree $p_{g}-2$ in $\mathbb{P}^{p_{g}-1}$. Then there are the following possibilities:

(1) $S$ is smooth and we have a factorization

$$
\phi_{1}: X \rightarrow X_{\mathrm{can}} \stackrel{\pi}{\rightarrow} S \hookrightarrow \mathbb{P}^{p_{g}-1} .
$$

The map $\pi$ is a finite flat morphism of degree 2 and $X_{\text {can }}$ has at worst $\mathrm{Du}$ Val singularities. If $\mathcal{L}$ denotes the invertible sheaf associated with $\pi$, then

- $S \cong \mathbb{P}^{2}, p_{g}=3$ and $\mathcal{L} \cong \mathcal{O}_{\mathbb{P}^{2}}(4)$

- $S \cong \mathbb{P}^{2}, p_{g}=6$ and $\mathcal{L} \cong \mathcal{O}_{\mathbb{P}^{2}}(5)$

- $S \cong \mathbb{F}_{d}, 0 \leq d \leq \min \left\{p_{g}-4, \frac{1}{2}\left(p_{g}+2\right)\right\}, p_{g}-d$ is even and $\mathcal{L} \cong \mathcal{O}_{\mathbb{F}_{d}}\left(3 \Delta_{0}+\frac{1}{2}\left(p_{g}+2+3 d\right) \Gamma\right)$.

(2) $S$ is the cone over a rational normal curve of degree $p_{g}-2$ in $\mathbb{P}^{p_{g}-1}$ with $4 \leq p_{g} \leq 6$. There exists a partial desingularization $X^{\prime}$ of $X_{\text {can }}$ such that $\phi_{1}$ factors as

$$
\phi_{1}: X \rightarrow X^{\prime} \stackrel{\pi}{\rightarrow} \mathbb{F}_{p_{g}-2} \rightarrow S \hookrightarrow \mathbb{P}^{p_{g}-1},
$$

where $\pi$ is finite and flat of degree 2. If $\mathcal{L}$ denotes the invertible sheaf associated with $\pi$, then $\mathcal{L} \cong \mathcal{O}_{\mathbb{F}_{p_{g}-2}}\left(3 \Delta_{0}+\left(2 p_{g}-2\right) \Gamma\right)$.

Proof. See [Lie2, Theorem 3.3] and [Lie2, Proposition 4.2]. 
Theorem 3.8. Let $X$ be an odd Horikawa surface with $p_{g} \geq 5$. Then the canonical linear system has a unique base point and its canonical image $S=\phi_{1}(X)$ is a surface of degree $p_{g}-2$ in $\mathbb{P}^{p_{g}-1}$. We denote by $\widetilde{X}$ the blow-up of $X$ in this base-point and obtain the following possibilities for the induced morphism:

(1) Case A: $S$ is smooth and we have a factorization

$$
\begin{aligned}
& \widetilde{\phi}_{1}: \tilde{X} \rightarrow \widetilde{X}^{\prime} \rightarrow S \hookrightarrow \mathbb{P}^{p_{g}-1} \\
& \searrow \underset{\tilde{X}^{\prime \prime}}{\stackrel{\pi}{\rightarrow}} \stackrel{\uparrow}{\widetilde{S}}
\end{aligned}
$$

where the upper row is the Stein factorization of $\widetilde{\phi}_{1}$. The morphism $\nu$ is the blow-up of $S$ in two (possibly infinitely near) points $x$, y lying on a fiber $\Gamma$ and with exceptional divisors $E_{x}, E_{y}$. The map $\pi$ is a finite flat morphism of degree 2 and $\widetilde{X}^{\prime \prime}$ has at worst Du Val singularities. If $\mathcal{L}$ denotes the invertible sheaf associated with $\pi$, then

$$
\widetilde{S} \cong \widetilde{\mathbb{F}}_{d} \quad \text { and } \quad \mathcal{L} \cong \mathcal{O}_{\widetilde{\mathbb{F}}_{d}}\left(3 \nu^{*} \Delta_{0}+\frac{1}{2}\left(p_{g}+4+3 d\right) \nu^{*} \Gamma-2 E_{x}-2 E_{y}\right)
$$

where $0 \leq d \leq \min \left\{p_{g}-4, \frac{1}{2}\left(p_{g}+1\right)\right\}$ and $p_{g}-d$ is even.

(2) Case $A^{\prime}: S$ is the cone over a rational normal curve. Then $p_{g}=5$ and $S \cong \mathbb{F}_{3}$, but $S \rightarrow \mathbb{P}^{4}$ is no longer an embedding but contracts $\Delta_{0}$. In this case, we have

$$
\widetilde{S} \cong \widetilde{\mathbb{F}}_{3} \quad \text { and } \quad \mathcal{L} \cong \mathcal{O}_{\widetilde{\mathbb{F}}_{3}}\left(3 \nu^{*} \Delta_{0}+9 \nu^{*} \Gamma-2 E_{x}-2 E_{y}\right)
$$

(3) Case $B_{1}$ : same as in case $A$ but only for $S \cong \mathbb{F}_{1}$ and $p_{g}=5$. Then $\nu$ is the blow-up of $S$ in a point $x \in \Delta_{0}$ and

$$
\widetilde{S} \cong \widetilde{\mathbb{F}}_{1} \quad \text { and } \quad \mathcal{L} \cong \mathcal{O}_{\widetilde{\mathbb{F}}_{1}}\left(4 \nu^{*} \Delta_{0}+5 \nu^{*} \Gamma-2 E_{x}\right)
$$

(4) Case $B_{2}$ : same as in case $A$ but only for $S \cong \mathbb{F}_{2}$ and $p_{g}=6$. Then no blow-up is needed and we have

$$
S=\widetilde{S} \cong \mathbb{F}_{2} \quad \text { and } \quad \mathcal{L} \cong \mathcal{O}_{\mathbb{F}_{2}}\left(4 \Delta_{0}+7 \Gamma\right) .
$$

Proof. For characteristic $\neq 2$ this is carried out in [Hor2, Section 1]. In characteristic 2 it also follows from loc.cit., only the standard facts about double covers have to be replaced by the characteristic 2 description, see Appendix $\mathrm{A}$ below. In order to get the bounds on $p_{g}$ and $d$ also in characteristic 2 we still can use [Hor2, Lemma 1.2], which is justified by [Lie2, Lemma 4.1].

Remark 3.9. The division into cases A and B goes back to [Hor2, Section 1].

From the explicit description of $\mathcal{L}$ in Theorem 3.7 and Theorem 3.8 we get the following vanishing results, which will be important later on

Lemma 3.10. Let $\mathcal{L}$ be an invertible sheaf as in Theorem 3.7 or Theorem 3.8 Then

$$
h^{1}\left(\mathcal{L}^{\otimes i}\right)=0 \quad \text { for all } \quad i \in \mathbb{Z} .
$$

Proof. For even Horikawa surfaces and odd Horikawa surfaces of type $B_{2}$ this is straight forward, e.g., using [Lie2, Lemma 3.5]. For the remaining odd Horikawa 
surfaces, let $\nu: \widetilde{S} \rightarrow S$ be the blow-up in $\{x, y\}$. We write $\mathcal{L}$ as

$$
\mathcal{L} \cong \nu^{*} \mathcal{M} \otimes \mathcal{O}_{\widetilde{S}}\left(-2 E_{x}-2 E_{y}\right) .
$$

We compute $h^{1}\left(\widetilde{S}, \nu^{*} \mathcal{M}^{\otimes i}\right)=h^{1}\left(S, \mathcal{M}^{\otimes i}\right)=0$ for all $i \in \mathbb{Z}$ as before. For $i \geq 0$ the theorem on formal functions gives $R^{1} \nu_{*}\left(\mathcal{L}^{\otimes i}\right)=0$, and then the GrothendieckLeray spectral sequence yields $h^{1}\left(\widetilde{S}, \mathcal{L}^{\otimes i}\right)=h^{1}\left(S, \nu_{*}\left(\mathcal{L}^{\otimes i}\right)\right)$. Denote by $Z_{i}$ the closed subscheme of $S$ corresponding to the ideal sheaf $\nu_{*} \mathcal{O}_{\widetilde{S}}\left(-2 i E_{x}-2 i E_{y}\right)$. Taking cohomology in

$$
\left.0 \rightarrow \nu_{*}\left(\mathcal{L}^{\otimes i}\right) \rightarrow \mathcal{M}^{\otimes i} \rightarrow \mathcal{M}^{\otimes i}\right|_{Z_{i}} \rightarrow 0
$$

a tedious check reveals that the boundary map $\delta: H^{0}\left(Z_{i}, \mathcal{M}^{\otimes i}\right) \rightarrow H^{1}\left(S, \nu_{*}\left(\mathcal{L}^{\otimes i}\right)\right)$. By the vanishing already obtained, we conclude $h^{1}\left(\widetilde{S}, \mathcal{L}^{\otimes i}\right)=0$ for $i \geq 0$.

Similarly, one proves $h^{1}\left(\widetilde{S}, \mathcal{L}^{\otimes-i} \otimes \omega_{\widetilde{S}}\right)=0$ for $i \leq 0$ and using Serre duality, we obtain $h^{1}\left(\widetilde{S}, \mathcal{L}^{\otimes i}\right)=0$ for $i \leq 0$.

Theorem 3.8 and Theorem 3.7 give the possible list of even Horikawa surfaces and odd Horikawa surfaces with $p_{g} \geq 5$ in arbitrary characteristic. It remains to prove their existence:

Theorem 3.11. All possible cases in Theorem 3.8 and Theorem 3.7 do exist in arbitrary characteristic. Moreover, in characteristic 2 all these types exist with inseparable as well as separable canonical maps.

PROOF. In characteristic $p \neq 2$, even Horikawa surfaces are easily constructed along the lines of [Hor1, Section 1] and odd Horikawa surfaces with $p_{g} \geq 5$ along the lines of [Hor2, Section 1].

We may thus assume $p=2$. A straight forward check shows that the sheaves $\mathcal{L}$ of Theorem 3.7 satisfy the assumptions of Theorem 2.4. This settles existence of even Horikawa surfaces with inseparable canonical map, which we have already obtained in [Lie2, Section 5].

Let $\Gamma_{0}$ be the fiber of $\mathbb{F}_{d} \rightarrow \mathbb{P}^{1}$ that contains the points $x, y$ in the statement of Theorem 3.8. Consider the subspace $V \subseteq H^{0}\left(\widetilde{S}, \mathcal{L}^{\otimes 2}\right)$ consisting of those sections that vanish along the strict transform $\widehat{\Gamma}$ of $\Gamma_{0}$ on $\widetilde{S}$. Assume furthermore that in case $A$ and $A^{\prime}$ the points $x, y$ are chosen as follows:

in case $A: \quad x, y \notin \Delta_{0} \quad$ if $\quad p_{g} \geq 3 d-3$,

in case $A: \quad x \in \Delta_{0}, y \notin \Delta_{0} \quad$ if $\quad 3 d-3>p_{g} \geq 2 d-1$,

in case $A^{\prime}: \quad x \in \Delta_{0}$.

A straight forward, yet tedious calculation reveals that the subspace $V$ fulfills the assumptions of Theorem 2.4 Note that $\pi^{*} \widehat{\Gamma}=2 E$ for some rational curve $E$ on the associated inseparable cover and thus $E^{2}=-1$. Hence $E$ is an exceptional $(-1)$-curve as in characteristic $\neq 2$. This settles existence of all cases of Theorem 3.8 with inseparable canonical map in characteristic 2 .

From Lemma 3.10 we get $\operatorname{ext}^{1}\left(\mathcal{L}^{\vee}, \mathcal{O}_{X}\right)=h^{1}(\mathcal{L})=0$ and so (9) splits. Thus, the double cover $\pi$ for a Horikawa surface is of the form $\pi: \operatorname{Spec}\left(\mathcal{O}_{\widetilde{S}} \oplus \mathcal{L}^{\vee}\right) \rightarrow \widetilde{S}$. The $\mathcal{O}_{\widetilde{S}}$-structure is globally given by $z^{2}+f z+s=0$ where $s$ is a global section of $\mathcal{L}^{\otimes 2}$. The canonical map is inseparable if and only if $f=0$. Since $h^{0}(\mathcal{L}) \neq 0$ in 
all cases, a generic global section $f$ of $\mathcal{L}$ yields a Horikawa surface with separable canonical map. We refer to [Lie2, Section 6] for technical details.

\section{Moduli SPaCeS}

In this section we give a characteristic-free description of moduli spaces of even Horikawa surfaces with fixed canonical image, which turn out to be irreducible and unirational. In characteristic 2, surfaces with inseparable canonical map form an irreducible, unirational, proper and closed subscheme inside these moduli spaces. We restrict ourselves to even Horikawa surfaces in order to keep our discussion short. The interested reader will have no difficulties working out the corresponding statements for odd Horikawa surfaces.

We obtained the case of separable canonical maps via a deformation argument from the inseparable ones. This is not a coincidence as we shall see now. To make this precise, let $S$ be a surface of minimal degree $n-2$ inside $\mathbb{P}^{n-1}$. Then we define the following sets

$$
\begin{array}{lll}
\mathcal{M}^{\text {even }}(S, n) & :=\quad \begin{array}{r}
\text { even Horikawa surfaces with } p_{g}=n \text { and } \\
\text { canonical image } S\} / \cong
\end{array} \\
\mathcal{M}^{\text {even,insep }}(S, n):=\quad\left\{\begin{array}{c}
\text { surfaces in } \left.\mathcal{M}^{\text {even }}(S, n) \text { with inseparable } \phi_{1}\right\}
\end{array}\right.
\end{array}
$$

Whether the set $\mathcal{M}^{\text {even }}(S, n)$ is empty or not is answered by Theorem 3.7 and Theorem 3.11. Here is a characteristic-free description of these spaces:

Theorem 4.1. Whenever $\mathcal{M}^{\text {even }}\left(S, p_{g}\right)$ is not empty it carries the structure of an irreducible and unirational scheme of dimension

$$
h^{0}\left(S, \mathcal{L}^{\otimes 2}\right)-\operatorname{dim} \operatorname{Aut}(S)-1,
$$

where $\mathcal{L}$ is as in Theorem 3.7 Whenever

- $S \cong \mathbb{P}^{2}$, which implies $p_{g}=3$ or $p_{g}=6$, or

- $S \cong \mathbb{F}_{d}$ and $p_{g} \geq 3 d-2$ or $p_{g}=2 d-2$, or

- $S$ is not smooth and $p_{g}=4$ or $p_{g}=6$,

then the generic surface of $\mathcal{M}^{\text {even }}\left(S, p_{g}\right)$ has a smooth canonical model.

In the remaining cases the generic canonical model has $A_{1}$-singularities. The number of $A_{1}$-singularities of the generic canonical model is as follows:

$$
\begin{array}{l||c|c}
\left(S, p_{g}\right) & \left(\mathbb{F}_{d}, p_{g}\right), 3 d-2>p_{g}>2 d-2 & (\text { cone }, 5) \\
\hline A_{1}-\text { singularities } & p_{g}+2-2 d & 1
\end{array}
$$

Proof. Assume that $S$ is smooth and let $X \in \mathcal{M}^{\text {even }}\left(S, p_{g}\right)$. From the vanishing $\operatorname{ext}^{1}\left(\mathcal{L}^{\vee}, \mathcal{O}_{S}\right)=h^{1}(\mathcal{L})=0$ by Lemma 3.10 it follows that $\pi: X_{\text {can }} \rightarrow S$ is isomorphic to $\pi: \operatorname{Spec}\left(\mathcal{O}_{S} \oplus \mathcal{L}^{\vee}\right) \rightarrow S$. The $\mathcal{O}_{S}$-algebra structure is given by $z^{2}+f z+g=0$ where $f \in H^{0}(S, \mathcal{L})$ and $g \in H^{0}\left(S, \mathcal{L}^{\otimes 2}\right)$.

Conversely, for generic choices of global sections of $\mathcal{L}$ and $\mathcal{L}^{\otimes 2}$, the associated double cover $\pi$ will be the canonical model of an even Horikawa surface with canonical image $S$ inside $\mathbb{P}^{p_{g}-1}$. In characteristic $\neq 2$ this is classical. In characteristic 2 the argument is as follows: a generic global section $g \in H^{0}\left(S, \mathcal{L}^{\otimes 2}\right)$ and $0=f \in H^{0}(S, \mathcal{L})$ give rise to an even Horikawa surface with the desired 
properties by Theorem 2.4 But then, fixing $g$, a generic choice of $f \in H^{0}(S, \mathcal{L})$ yields again a surface with at worst Du Val singularities [Lie2, Proposition 6.1], i.e., the canonical model of an even Horikawa surface with the desired properties. Thus, the assertion is also true in characteristic 2 .

Therefore, there exists an open and dense subset $U \subseteq H^{0}(S, \mathcal{L}) \oplus H^{0}\left(S, \mathcal{L}^{\otimes 2}\right)$ mapping surjectively onto $\mathcal{M}^{\text {even }}\left(S, p_{g}\right)$. Now, suppose we are given two $\mathcal{O}_{S^{-}}$ algebras $\mathcal{O}_{S}\left[z_{i}\right] /\left(z_{i}^{2}+f_{i} z_{i}+g_{i}\right)$ that are isomorphic over $S$. A straight forward computation shows that such an isomorphism is given by $z_{1} \mapsto a \cdot z_{2}+h$ for some $a \in k^{\times}$and $h \in H^{0}(S, \mathcal{L})$. Conversely, such a map defines an isomorphism over $S$. Thus, given two isomorphic even Horikawa surfaces, there exists an isomorphism of $S$ after which they become isomorphic as surfaces over $S$. Thus, $\mathcal{M}^{\text {even }}\left(S, p_{g}\right)$ arises from $U$ by quotienting out the indicated actions of $\operatorname{Aut}(S)$, $\mathbb{G}_{m}$ and $H^{0}(S, \mathcal{L})$. In particular, $\mathcal{M}^{\text {even }}\left(S, p_{g}\right)$ is irreducible, unirational and of the stated dimension.

In case $S$ is a cone the arguments are similar and left to the reader.

Finally, if $S=\mathbb{P}^{2}, S=\mathbb{F}_{d}$ and $p_{g}>3 d-2$ or $p_{g}=4$ and $S$ is a cone then $\mathcal{L}$ and $\mathcal{L}^{\otimes 2}$ are very ample. In characteristic $\neq 2$ we can thus choose $0=f \in H^{0}(S, \mathcal{L})$ and $g \in H^{0}\left(S, \mathcal{L}^{\otimes 2}\right)$ with smooth zero divisor and the associated double cover will be smooth. In characteristic 2 this conclusion still holds true by Theorem 2.6 In the remaining cases, sections of $\mathcal{L}$ and $\mathcal{L}^{\otimes 2}$ necessarily vanish along $\Delta_{0}$. We leave it to the reader that in case $S=\mathbb{F}_{d}$ and $p_{g}=3 d-2$ and $S$ a cone and $p_{g}=6$ it is still possible to find smooth sections whereas this is impossible in the other cases.

As to the number of $A_{1}$-singularities, let us first deal with $S=\mathbb{F}_{d}$ and $3 d-2>$ $p_{g}>2 d-2$. In characteristic $p \neq 2$, a generic section of $\mathcal{L}^{\otimes 2}$ vanishes along the union of $\Delta_{0}$ and a smooth divisor intersecting $\Delta_{0}$ transversally in $p_{g}+2-2 d$ points. Thus, the associated double cover has the claimed number of $A_{1}$-singularities. In characteristic 2 a similar argument and Theorem 2.4 reveal that an inseparable cover associated to a generic section of $\mathcal{L}^{\otimes 2}$ has at least $A_{1}$ singularities at $p_{g}+$ $2-2 d$ points lying on $\Delta_{0}$ (but in fact more, see Theorem 4.3 below). A generic section of $\mathcal{L}$ vanishes along $\Delta_{0}$ and a smooth divisor intersecting transversally. This and a local computation reveals that a generic Artin-Schreier covering still has $p_{g}+2-2 d$ singularities of type $A_{1}$. We leave the details and the case where $S$ is a cone to the reader.

Remark 4.2. Since $S$ and $\mathcal{L}$ are defined over the integers, the proof provides an explicit construction of $\mathcal{M}^{\text {even }}\left(S, p_{g}\right)$ over Spec $\mathbb{Z}$. See also Theorem 7.3 below.

We included the statement about generic canonical models being smooth or not for the following reason: in case the canonical map is inseparable the canonical model has always singularities:

Theorem 4.3. Suppose that we are in characteristic 2 and that $\mathcal{M}^{\mathrm{even}}\left(S, p_{g}\right)$ is not empty. Then the subset

$$
\mathcal{M}^{\text {even,insep }}\left(S, p_{g}\right) \quad \subseteq \quad \mathcal{M}^{\text {even }}\left(S, p_{g}\right)
$$

forms an irreducible and unirational subscheme of codimension $h^{0}(S, \mathcal{L})$. 
Every surface of $\mathcal{M}^{\text {even,insep }}\left(S, p_{g}\right)$ has a singular canonical model, but the generic member has only $\mathrm{Du}$ Val singularities of type $A_{1}$. The number of $A_{1}$ singularities of the generic canonical model is as follows:

\begin{tabular}{l|cccc}
$\left(S, p_{g}\right)$ & $\left(\mathbb{P}^{2}, 3\right)$ & $\left(\mathbb{P}^{2}, 6\right)$ & $\left(\mathbb{F}_{d}, p_{g}\right)$ & $\left(\right.$ cone,$\left.p_{g}\right)$ \\
\hline$A_{1}-$ singularities & 43 & 73 & $12+10 p_{g}$ & $12+10 p_{g}$
\end{tabular}

In characteristic $\neq 2$, the set $\mathcal{M}^{\text {even,insep }}\left(S, p_{g}\right)$ is empty.

PROOF. The canonical map of an even Horikawa surface is generically finite of degree 2 and so $\mathcal{M}^{\text {even,insep }}\left(S, p_{g}\right)$ is empty in characteristic $\neq 2$. In characteristic 2, surfaces with inseparable canonical map correspond to those $\mathcal{O}_{S}$-algebras $\mathcal{O}_{S}[z] /\left(z^{2}+f z+g\right)$, where $0=f \in H^{0}(S, \mathcal{L})$, keeping the notations of the proof of Theorem 4.1 By the arguments given there we find an open dense subset of $H^{0}\left(S, \mathcal{L}^{\otimes 2}\right)$ mapping surjectively onto $\mathcal{M}^{\text {even,insep }}\left(S, p_{g}\right)$. Taking into account the actions of $H^{0}(S, \mathcal{L}), \mathbb{G}_{m}$ and $\operatorname{Aut}(S)$ that link isomorphic surfaces we conclude as before that $\mathcal{M}^{\text {even,insep }}\left(S, p_{g}\right)$ is irreducible, unirational and obtain the asserted codimension.

Since $c_{2}\left(\Omega_{S} \otimes \mathcal{L}^{\otimes 2}\right)$ is non-zero in all cases the inseparable double covers can never be smooth, see Section 2 . However, by Theorem 2.4 the singularities of a generic member of $\mathcal{M}^{\text {even,insep }}\left(S, p_{g}\right)$ are Du Val singularities of type $A_{1}$.

In order to determine the number of $A_{1}$ singularities let $\pi: X_{\text {can }} \rightarrow S$ be the canonical model and its inseparable double cover, and assume $S$ is smooth. Let $\widetilde{\pi}: X \rightarrow \widetilde{S}$ be the canonical resolution of singularities as given by Proposition A.2. The singularities of $X_{\text {can }}$ all being of type $A_{1}$, the number of these singularities equals the number of blow-ups in closed points $\widetilde{S} \rightarrow S$, which is equal to $b_{2}(\widetilde{S})-$ $b_{2}(S)$. On the other hand, $\widetilde{\pi}$ is finite and purely inseparable, and $X$ and $\widetilde{S}$ are smooth, which implies $b_{2}(X)=b_{2}(\widetilde{S})$. Since $X$ is an even Horikawa surface we have $b_{2}(X)+2=c_{2}(X)=12 \chi\left(\mathcal{O}_{X}\right)-K^{2}$ from which it is easy to compute the stated numbers. In case $S$ is not smooth we have to blow up once to start with and conclude as before.

Remarks 4.4. We note

(1) Even if every canonical model of $\mathcal{M}^{\text {even }}\left(S, p_{g}\right)$ in characteristic 2 is singular, the number of singularities of a generic member of $\mathcal{M}^{\text {even }}\left(S, p_{g}\right)$ is less than that of a generic member of $\mathcal{M}^{\text {even,insep }}\left(S, p_{g}\right)$.

(2) The explicit examples of even Horikawa surfaces with inseparable canonical map of [Lie2, Sections 5 and 6] have $D_{4}$-singularities and are thus rather special. 


\section{SMALL INVARIANTS}

We are left with the description of odd Horikawa surfaces with $p_{g} \leq 4$. In this case the canonical map need no longer be generically finite of degree 2 onto a surface of minimal degree and new cases show up:

Proposition 5.1. Let $X$ be an odd Horikawa surface with $p_{g} \leq 4$. Then we have the following cases

(1) $p_{g}=2, K_{X}^{2}=1$ and the canonical map is a pencil.

(2) $p_{g}=3, K_{X}^{2}=3$, the canonical linear system has a unique base point. After blowing it up we obtain a generically finite morphism of degree 2 onto $\mathbb{P}^{2}$.

(3) $p_{g}=3, K_{X}^{2}=3$, the canonical linear system has no basepoints and defines a generically finite morphism of degree 3 onto $\mathbb{P}^{2}$.

(4) $p_{g}=4, K_{X}^{2}=5$, the canonical linear system has no basepoints and defines a birational morphism onto a quintic surface in $\mathbb{P}^{3}$.

(5) $p_{g}=4, K_{X}^{2}=5$, the canonical linear system has a unique basepoint. After blowing it up we obtain a generically finite morphism of degree 2 onto a (possibly singular) quadric surface in $\mathbb{P}^{3}$.

PROOF. If $p_{g}=2$ then the canonical map defines a pencil, whereas for $p_{g} \geq 3$ the canonical image is always a surface by [Lie2, Theorem 2.1]. As in the proof of Proposition 3.2 the result follows from Theorem 1.4 .

Surfaces with $\mathbf{p}_{\mathrm{g}}=\mathbf{2}$ and $\mathbf{K}_{\mathrm{X}}^{2}=1$. These surfaces have the smallest invariants possible among all surfaces on the Noether lines. The canonical map defines a pencil, which does not happen for surfaces on the Noether lines with larger invariants. We use the bicanonical map to obtain an explicit classification.

Theorem 5.2. Let $X$ be a minimal surface of general type with $p_{g}=2$ and $K^{2}=$ 1. Then the bicanonical map is a morphism and factors as

$$
\phi_{\left|2 K_{X}\right|}: X \rightarrow X_{\text {can }} \stackrel{\pi}{\rightarrow} \mathbb{F}_{2} \stackrel{\nu}{\rightarrow} \mathbb{P}^{3},
$$

where $\pi$ is a finite flat morphism of degree 2 onto the Hirzebruch surface $\mathbb{F}_{2}$ and with associated invertible sheaf $\mathcal{L} \cong \mathcal{O}_{\mathbb{F}_{2}}\left(3 \Delta_{0}+5 \Gamma\right)$. The map $\nu$ contracts the (-2)-curve $\Delta_{0}$ on $\mathbb{F}_{2}$ and maps $\mathbb{F}_{2}$ onto a quadric cone in $\mathbb{P}^{3}$. Moreover, $h^{1}\left(\mathcal{O}_{X}\right)=0$ and the surface is algebraically simply connected.

In characteristic 2, such surfaces exist with separable as well as inseparable bicanonical maps.

PROOF. Ekedahl's inequality [Ek2, Corollary II.1.8] yields $h^{1}\left(\mathcal{O}_{X}\right) \leq 1$. Thus, we obtain $\chi\left(\mathcal{O}_{X}\right) \geq 2$ and so $h^{1}\left(\omega_{X}^{\vee}\right)=0$ by [Ek2, Corollary II.1.8]. In particular, $h^{0}\left(\omega_{X}^{\otimes 2}\right)=3$. Arguing as in [Hor2, Lemma 2.1], we conclude that the bicanonical linear system defines a morphism of degree 2 onto a quadric cone in $\mathbb{P}^{3}$. As in [Hor2, Section 2] we obtain the structure result of these surfaces. From the vanishing $h^{1}\left(\mathcal{L}^{\vee}\right)=0$ and (9) we conclude $h^{1}\left(\mathcal{O}_{X}\right)=0$ and the surface is algebraically simply connected by Lemma 3.4 
The existence of such surfaces in characteristic $\neq 2$ is shown along the lines of [Hor2, Section 2]. Surfaces with inseparable canonical maps arise as $\alpha_{\mathcal{L}}$-torsors over $\mathbb{F}_{2}$ and applying Theorem 2.4. Deforming these surfaces, we obtain surfaces with separable bicanonical maps.

Surfaces with $\mathbf{p}_{\mathrm{g}}=\mathbf{3}$ and $\mathbf{K}_{\mathbf{X}}^{2}=\mathbf{3}$. Here there are two cases and both do exist. Also, we encounter possibly inseparable canonical maps in characteristic 3.

Theorem 5.3. Let $X$ be a minimal surface of general type with $p_{g}=K^{2}=3$. Then

(1) either the canonical map has a unique base point and after blowing it up we obtain a morphism of degree 2 onto $\mathbb{P}^{2}$.

In characteristic 2 , such surfaces exist with separable as well as inseparable canonical maps.

(2) or the canonical map is a generically finite morphism of degree 3 onto $\mathbb{P}^{2}$.

In characteristic 3 , such surfaces exist with separable as well as inseparable canonical maps.

In any case, $h^{1}\left(\mathcal{O}_{X}\right)=0$ and the surface is algebraically simply connected.

ProOF. By Proposition 5.1 there can only be these two cases.

In case the canonical map has a unique base point, let $\widetilde{X} \rightarrow X$ be the blow-up of this base point and the canonical map extends to a generically finite morphism $\widetilde{\phi}_{1}: \widetilde{X} \rightarrow \mathbb{P}^{2}$. As explained in [Hor2, Section 2], the exceptional (-1)-curve of $\widetilde{X}$ maps to a line $\ell$ in $\mathbb{P}^{2}$ and $\widetilde{X}$ determines three points $x, y, z$ on $\ell$. Let $q: \widetilde{\mathbb{P}^{2}} \rightarrow \mathbb{P}^{2}$ be the blow-up in these three points with exceptional divisors $E_{x}, E_{y}$ and $E_{z}$. Then $\widetilde{\phi_{1}}$ factors over $\widetilde{\mathrm{P}^{2}}$. Let $\widetilde{X} \rightarrow X^{\prime} \rightarrow \widetilde{\mathrm{P}^{2}}$ be the Stein factorization. Then $X^{\prime} \rightarrow \widetilde{\mathrm{P}^{2}}$ is a finite flat double cover with associated invertible sheaf $\mathcal{L}^{\vee}=q^{*} \mathcal{O}_{\mathbb{P}^{2}}(-5) \otimes$ $\mathcal{O}_{\widetilde{\mathbb{P}^{2}}}\left(2 E_{x}+2 E_{y}+2 E_{z}\right)$. From the vanishing $h^{1}(\mathcal{L})=0$ we get existence as in Section 4. Moreover, from $h^{1}\left(\mathcal{L}^{\vee}\right)=0$ we conclude $h^{1}\left(\mathcal{O}_{X}\right)=0$ and thus, $X$ is algebraically simply connected by Lemma 3.4

In case the canonical map is a morphism of degree 3 we can factor it as $X \rightarrow$ $X_{\text {can }} \rightarrow \mathbb{P}^{2}$, where $X_{\text {can }}$ is the canonical model and $X_{\text {can }} \rightarrow \mathbb{P}^{2}$ is finite and flat of degree 3 . If $h^{1}\left(\mathcal{O}_{X}\right)=0$ then $X$ is algebraically simply connected. Moreover, in this case one can argue as in [Hor2, Section 2] to conclude that $X_{\text {can }}$ embeds as a hypersurface given by an equation $\psi^{3}+A \psi^{2}+B \psi+C=0$ into the vector bundle $V\left(\mathcal{O}_{\mathbb{P}^{2}}(2)\right) \rightarrow \mathbb{P}^{2}$. Here, $A, B, C$ are elements of $\operatorname{Sym}^{i} H^{0}\left(\omega_{X}\right)$ with $i=2,4,6$. Existence in characteristic $\neq 3$ follows as in loc. cit. Surfaces with inseparable

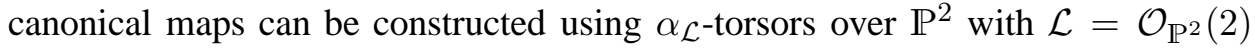
and applying Theorem 2.4. Deforming these surfaces, we obtain surfaces with separable canonical maps.

It remains to exclude the existence of surfaces whose canonical map is a morphism of degree 3 and $h^{1}\left(\mathcal{O}_{X}\right) \neq 0$. As in the proof of Proposition 3.5 such surfaces fulfill $h^{1}\left(\mathcal{O}_{X}\right)=1$ and we obtain $\chi\left(\mathcal{O}_{X}\right)=3$. Then $\pi: X_{\text {can }} \rightarrow \mathbb{P}^{2}$ is a finite flat morphism of degree 3 and we obtain a short exact sequence

$$
0 \rightarrow \mathcal{O}_{\mathbb{P}^{2}} \rightarrow \pi_{*} \mathcal{O}_{X_{\text {can }}} \rightarrow \mathcal{E} \rightarrow 0,
$$


where $\mathcal{E}$ is a locally free sheaf of rank 2 on $\mathbb{P}^{2}$. From $K^{2}=3$ and $\chi\left(\mathcal{O}_{X}\right)=3$ we get two equations for the Chern classes of $\mathcal{E}$, see [Pa, Proposition 8.1] and [Pa, Corollary 8.3]. A straight forward computation shows that these equations cannot be fulfilled by any locally free sheaf of rank 2 on $\mathbb{P}^{2}$. Thus $\mathcal{E}$ and $X$ do not exist.

Surfaces with $\mathbf{p}_{\mathrm{g}}=\mathbf{4}$ and $\mathbf{K}_{\mathrm{X}}^{2}=\mathbf{5}$. Again, we have to deal with two cases. We note that this class of Horikawa surfaces also fulfills $K^{2}=3 p_{g}-7$. Thus, Proposition 5.1 overlaps with the class of surfaces described in Theorem 1.5 .

Theorem 5.4. Let $X$ be a minimal surface of general type with $p_{g}=4$ and $K_{X}^{2}=$ 5. Then

(1) either the canonical map is a birational morphism that embeds the canonical model $X_{\text {can }}$ as a quintic surface into $\mathbb{P}^{3}$,

(2) or the canonical map has a unique base point. After blowing it up we obtain a morphism of degree 2 onto a possibly singular quadric surface in $\mathbb{P}^{3}$.

In characteristic 2 , such surfaces exist with separable as well as inseparable canonical maps.

In any case, $h^{1}\left(\mathcal{O}_{X}\right)=0$ and the surface is algebraically simply connected.

Proof. By Proposition 5.1 (or Theorem 1.5) there can only be these two cases.

In the first case, Theorem 1.5 implies that $K_{X}$ is very ample on $X_{\text {can }}$, embedding it as a quintic surface in $\mathbb{P}^{3}$. Since quintic surfaces in $\mathbb{P}^{3}$ with at worst Du Val singularities fulfill $h^{1}=0$ and are algebraically simply connected, the same is true for $X$.

In the second case, Proposition 5.1yields their structure. Thus, we have to consider double covers of $\mathbb{P}^{1} \times \mathbb{P}^{1}$ with associated invertible sheaf $\mathcal{L} \cong \mathcal{O}_{\mathbb{P}^{1} \times \mathbb{P}^{1}}(3,3)$, or of $\mathbb{F}_{2}$ with $\mathcal{L} \cong \mathcal{O}_{\mathbb{F}_{2}}\left(3 \Delta_{0}+3 \Gamma\right)$. From $h^{1}\left(\mathcal{L}^{\vee}\right)=0$ and (9) we deduce $h^{1}\left(\mathcal{O}_{X}\right)=0$ and hence these surfaces are algebraically simply connected by Lemma 3.4 Existence is shown as in Section 4.

\section{Hodge DEGENERATION AND CRYSTALLINE COHOMOLOGY}

In this section we show that that the Frölicher spectral sequence for surfaces on the Noether lines always degenerates at $E_{1}$. Moreover, we show that their Hodgeand Betti- numbers coincide. In particular, their crystalline cohomology groups are torsion-free. On the other hand, we show that there exist even Horikawa surfaces in arbitrary large characteristics, whose slope spectral sequence from Hodge-Wittto crystalline cohomology does not degenerate at $E_{1}$.

Proposition 6.1. Let $X$ be a surface on the Noether lines. Then $H^{0}\left(X, \Omega_{X}^{1}\right)=0$. In particular, all global 1-forms are d-closed.

PROOF. We will only deal with the cases where the canonical or bicanonical map is generically finite of degree 2 and leave quintic surfaces in $\mathbb{P}^{3}$ (which satisfy $p_{g}=4, K^{2}=5$ ) and triple covers of $\mathbb{P}^{2}$ with $p_{g}=K^{2}=3$ to the reader. 
Then, by our explicit classification there exists a birational model $X^{\prime}$ of $X$ with at worst Du Val singularities and a finite flat morphism $\pi: X^{\prime} \rightarrow S$ of degree 2 onto a smooth surface $S$. Let $\mathcal{L}$ be the associated invertible sheaf. By Lemma 3.10, we have $\operatorname{Ext}^{1}\left(\mathcal{L}^{\vee}, \mathcal{O}_{S}\right)=H^{1}(S, \mathcal{L})=0$, which implies that the exact sequence (9) splits. Thus, $X^{\prime}$ embeds into the total space of the vector bundle

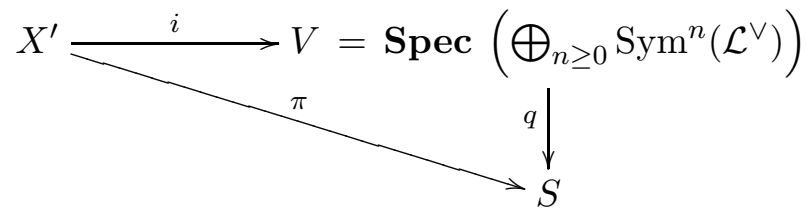

The cotangent sequence for $q$ on $V$ exhibits $\Omega_{V}^{1}$ as an extension of $q^{*}\left(\mathcal{L}^{\vee}\right)$ by $q^{*} \Omega_{S}^{1}$. In particular, $H^{0}\left(V, \Omega_{V}^{1}\right)=0$. Passing to reflexive hulls, i.e., taking double duals, the conormal sequence becomes

$$
0 \rightarrow \mathcal{N}_{X / V}^{\vee \vee} \rightarrow i^{*}\left(\Omega_{V}^{1}\right) \rightarrow\left(\Omega_{X^{\prime}}^{1}\right)^{\vee \vee} \rightarrow 0,
$$

where injectivity on the left follows from torsion-freeness. Note that $\operatorname{det}\left(\left(\Omega_{X^{\prime}}^{1}\right)^{\vee \vee}\right)$ and $\omega_{X^{\prime}}$ are isomorphic, since they coincide outside codimension two and are both reflexive sheaves on a normal variety. Taking determinants in (8) and using $\omega_{X^{\prime}} \cong \pi^{*}\left(\omega_{S} \otimes \mathcal{L}\right)$ we see that $\mathcal{N}_{X^{\prime} / V}^{\vee V}$ is isomorphic to $\pi^{*}\left(\mathcal{L}^{-2}\right)$. Pushing $\mathcal{N}_{X^{\prime} / V}^{\vee \vee}$ down to $S$, and using $H^{1}\left(S, \mathcal{L}^{-2}\right)=H^{1}\left(S, \mathcal{L}^{-3}\right)=0$ from Lemma 3.10 . we conclude $H^{1}\left(X^{\prime}, \mathcal{N}_{X^{\prime} / V}^{\vee \vee}\right)=0$. Thus, taking cohomology in (8) we obtain $H^{0}\left(X^{\prime},\left(\Omega_{X^{\prime}}^{1}\right)^{\vee \vee}\right)=0$.

Now, let $\nu: \widetilde{X} \rightarrow X^{\prime}$ be a resolution of singularities with exceptional divisor $Z$ and and let $U$ be the smooth locus of $X^{\prime}$. By reflexivity we have $H^{0}\left(U, \Omega_{U}^{1}\right)=$ $H^{0}\left(X^{\prime},\left(\Omega_{X^{\prime}}^{1}\right)^{\vee \vee}\right)=0$. On the other hand, since $\nu$ is the resolution of Du Val singularities there are never global sections of $\Omega_{\widetilde{X}}^{1}$ entirely supported on $Z$ (in fact, global sections of $\Omega_{\widetilde{X}}^{1}$ vanish along $\left.Z\right)$. Thus, the restriction map $H^{0}\left(\widetilde{X}, \Omega_{\widetilde{X}}^{1}\right) \rightarrow$ $H^{0}\left(U, \Omega_{U}^{1}\right)$ is injective and we conclude $H^{0}\left(X, \Omega_{X}^{1}\right)=H^{0}\left(\widetilde{X}, \Omega_{\widetilde{X}}^{1}\right)=0$.

Let us recall that the Frölicher spectral sequence

$$
E_{1}^{p, q}=H^{q}\left(X, \Omega_{X}^{p}\right) \Rightarrow H_{\mathrm{dR}}^{p+q}(X)
$$

links Hodge cohomology to deRham cohomology of smooth projective varieties. Over fields of characteristic zero, as well as for curves and Abelian varieties over arbitrary ground fields, this spectral sequence degenerates at $E_{1}$.

Let us also recall, e.g. from [IIl, II.(4.9.1)], the universal coefficient formula for crystalline cohomology:

$$
0 \rightarrow H_{\text {cris }}^{i}(X / W) \otimes_{W} k \rightarrow H_{\mathrm{dR}}^{i}(X / k) \rightarrow \operatorname{Tor}_{1}^{W}\left(H_{\text {cris }}^{i+1}(X / W), k\right) \rightarrow 0 .
$$

It shows that deRham- and Betti-numbers coincide if and only if all crystalline cohomology groups are torsion-free.

Theorem 6.2. Let $X$ be a surface on the Noether lines. Then

(1) its Frölicher spectral sequence degenerates at $E_{1}$-level, and 
(2) all crystalline cohomology groups are torsion-free.

In particular, the deRham- and Betti-numbers coincide.

Proof. We have $h^{10}=h^{01}=0$ for surfaces on the Noether lines by the classification results of the previous sections and Proposition 6.1. Moreover, we get $h^{12}=h^{21}=0$ from Serre duality. Already the existence of the Frölicher spectral sequence gives the inequalities $h_{\mathrm{dR}}^{1} \leq h^{01}+h^{10}$ and $h_{\mathrm{dR}}^{3} \leq h^{21}+h^{12}$. Thus, we obtain $h_{\mathrm{dR}}^{1}=h_{\mathrm{dR}}^{3}=0$. Moreover, $\sum_{n}(-1) h_{\mathrm{dR}}^{n}$ coincides with $\sum_{i+j}(-1)^{i+j} h^{i j}$, which implies $h_{\mathrm{dR}}^{2}=h^{02}+h^{11}+h^{20}$. These equalities prove degeneration of the Frölicher spectral sequence at $E_{1}$.

From $h_{\mathrm{dR}}^{1}=h_{\mathrm{dR}}^{3}=0$ and the universal coefficient formula for crystalline cohomology we obtain $H_{\text {cris }}^{1}=H_{\text {cris }}^{3}=0$ and see that $H_{\text {cris }}^{2}$ is torsion-free.

In [IIl] the Hodge-Witt cohomology groups $H^{j}\left(X, W \Omega_{X}^{i}\right)$ are constructed. For smooth projective varieties, these are $W=W(k)$-modules of finite rank, whose torsion-groups may not be finitely generated. The slope spectral sequence

$$
E_{1}^{p, q}=H^{q}\left(X, W \Omega_{X}^{p}\right) \Rightarrow H_{\text {cris }}^{p+q}(X / W)
$$

links Hodge-Witt- to crystalline cohomology. For surfaces, it degenerates at $E_{1}$ if and only if $H^{2}\left(X, W \mathcal{O}_{X}\right)$ is finitely generated [IIl, Corollaire II.3.14]. Moreover, it degenerates always modulo torsion at $E_{1}$, see [IIl, Théorème II.3.2].

In our case, this finite generation is closely related to the formal Brauer group: namely, if $X$ is a surface on the Noether lines then $h^{01}=h^{03}=0$ for dimensional reasons and by the classification results of the previous sections. Thus, the functor on Artin-algebras over $k$

$$
\operatorname{Br}: A \mapsto \operatorname{ker}\left(H^{2}\left(X \times_{k} A, \mathbb{G}_{m}\right) \rightarrow H^{2}\left(X, \mathbb{G}_{m}\right)\right)
$$

is pro-representable by a smooth formal group $\widehat{\operatorname{Br}}(X)$ of dimension $p_{g}=h^{02}$, the formal Brauer group. Moreover, its Dieudonné module of typical curves can be identified with $H^{2}\left(X, W \mathcal{O}_{X}\right)$. In particular, it is finitely generated if and only if $\widehat{\mathrm{Br}}(X)$ is of finite height, i.e., has no unipotent part [AM, Section II.4].

In order to show that $H^{2}\left(X, W \mathcal{O}_{X}\right)$ may not be finitely generated for surfaces on the Noether lines in arbitrary large characteristics, let us recall the following result [LS, Theorem 5.4]:

Theorem 6.3 (-,Schütt). There exists an arithmetic progression $P$ of primes of density at least 0.99999985 such that for all $p \in P$ there exists an even Horikawa surface $X_{p}$ in characteristic $p$ that is unirational.

For such unirational surfaces $\widehat{\operatorname{Br}}(X)$ is unipotent [IIl, Remarque II.5.13]. Thus:

Corollary 6.4. For all $p \in P$ there exists an even Horikawa surface $X_{p}$ in characteristic $p$, such that $H^{2}\left(X_{p}, W \mathcal{O}_{X_{p}}\right)$ is not finitely generated. In particular, its slope spectral sequence does not degenerate at $E_{1}$-level. 


\section{LIFTING AND TOPOLOGICAL FLATNESS OF MODULI SPACES}

In this section we show that the canonical model of a surface on the Noether lines lifts over the Witt ring $W(k)$. Its minimal model lifts via an algebraic space over possibly ramified extensions of $W(k)$. Finally, we use these lifting results to prove that moduli spaces of surfaces on the Noether lines are topologically flat over the integers.

We start with a more general observation: let $k$ be a field of characteristic $p>0$. We choose a $\operatorname{DVR}(R, \mathfrak{m})$ of characteristic zero with residue field $R / \mathfrak{m} \cong k$ and denote its quotient field by $K$. Let us also assume that $R$ is a Nagata ring. By a lifting of a variety $X \rightarrow$ Spec $k$ over $R$ we mean a scheme (or an algebraic space if explicitly stated) that is flat over $\operatorname{Spec} R$ with special fiber $X$.

Proposition 7.1. Let $X \rightarrow$ Spec $k$ be a surface of general type, not necessarily minimal and with at worst Du Val singularities. If $X$ lifts over $R$ then

(1) its canonical model $X_{\text {can }}$ also lifts over $R$, and

(2) there exists a possibly ramified extension $R^{\prime} \supseteq R$ and an algebraic space, flat over $R^{\prime}$, with special fiber the minimal smooth model $X_{\min }$ of $X$.

Proof. Let $\mathcal{X} \rightarrow$ Spec $R$ be a lifting of $X$. Denote by $K$ the field of fractions of $R$ and by $\mathcal{X}_{K}$ the generic fiber of $\mathcal{X}$. Since $X$ and $R$ are Gorenstein, the same is true for $\mathcal{X}$ and $\mathcal{X}_{K}$. We denote by $\omega_{\mathcal{X}}$ its dualizing sheaf and note its restriction to the generic and special fiber yields the respective dualizing sheaves. Note that $\mathcal{X}_{K}$ also has at worst $\mathrm{Du}$ Val singularities [Lie2, Proposition 6.1].

Since $\mathcal{X}$ is flat over $R$ we have $\chi:=\chi\left(\mathcal{O}_{X}\right)=\chi\left(\mathcal{O}_{\mathcal{X}_{K}}\right)$ and $K^{2}:=K_{X}^{2}=$ $K_{\mathcal{X}_{K}}^{2}$. Moreover, we have $h^{1}\left(X, \omega_{X}^{\otimes n}\right)=0$ for $n \geq 3$ by [Ek2, Theorem II.1.7] and thus Riemann-Roch yields

$$
h^{0}\left(X, \omega_{X}^{\otimes n}\right)=\frac{n(n-1)}{2} K^{2}+\chi=h^{0}\left(\mathcal{X}_{K}, \omega_{\mathcal{X}_{K}}^{\otimes n}\right) \quad \text { for all } \quad n \geq 3 .
$$

This implies that for $n \geq 3$ global sections of $\omega_{X}^{\otimes n}$ lift to global sections of $\omega_{\mathcal{X}}^{\otimes n}$ and that the $H^{0}\left(\mathcal{X}, \omega_{\mathcal{X}}^{\otimes n}\right)$ are torsion-free $R$-modules [Hart, Corollary III.12.9]. Thus, the $n$-fold Veronese map for some $n \geq 3$ of the relative canonical ring

$$
\mathcal{X}_{\text {can }}^{(n)}:=\operatorname{Proj} \bigoplus_{k=0}^{\infty} H^{0}\left(\mathcal{X}, \omega_{\mathcal{X}}^{\otimes n k}\right) \rightarrow \operatorname{Spec} R
$$

is a projective scheme over $R$ with special (resp. generic) fiber isomorphic to the canonical model of $X$ (resp. $\mathcal{X}_{K}$ ). This gives the stated lifting of $X_{\text {can }}$.

By the main result of [Ar] there exists a possibly ramified extension $R^{\prime}$ of $R$ and an algebraic space over $\operatorname{Spec} R^{\prime}$ over which $\mathcal{X}_{\text {can }}^{(n)} \rightarrow \operatorname{Spec} R$ admits a simultaneous and minimal resolution of singularities. This gives the desired lifting of $X_{\min }$.

Using the explicit classification results of the previous sections we obtain

Theorem 7.2. Let $X$ be a surface on the Noether lines over an algebraically closed field $k$ of positive characteristic. Then

(1) the canonical model $X_{\text {can }}$ lifts over $W(k)$ 
(2) the minimal smooth model $X_{\min }$ lifts via an algebraic space over a possibly ramified extension of $W(k)$.

Proof. We only deal with the case that $X$ is an even Horikawa surface or an odd Horikawa surface with $p_{g} \geq 5$ and leave the remaining cases to the reader.

From Theorem 3.7 and Theorem 3.8 we see that there exists a surface $X^{\prime}$, birational to $X$, with at worst $\mathrm{Du}$ Val singularities and a finite flat morphism $\pi: X^{\prime} \rightarrow S$, where $S$ is a smooth rational surface. As usual, we denote by $\mathcal{L}$ the invertible sheaf associated to $\pi$. From the vanishing $\operatorname{ext}^{1}\left(\mathcal{L}^{\vee}, \mathcal{O}_{S}\right)=h^{1}(\mathcal{L})=0$ (Lemma 3.10) we see that $X^{\prime} \cong \operatorname{Spec}\left(\mathcal{O}_{S} \oplus \mathcal{L}^{\vee}\right)$ as $S$-scheme.

As already discussed in [Lie2, Section 7], Grothendieck's existence theorem implies that rational surfaces, and in particular $S$, lift over $W(k)$. Moreover, invertible sheaves on rational surfaces even lift uniquely. We can lift sections of these invertible sheaves if their $h^{1}$ vanishes [Lie2, Lemma 7.1]. This being the case for $\mathcal{L}$ and $\mathcal{L}^{\otimes 2}$ by Lemma 3.10, it follows that the whole double cover $\pi: X^{\prime}=\operatorname{Spec}\left(\mathcal{O}_{S} \oplus \mathcal{L}^{\vee}\right) \rightarrow S$ lifts over $W(k)$.

The result now follows from Proposition 7.1.

Let us recall that a scheme over a regular, integral, one-dimensional base scheme is called topologically flat if its generic fiber is dense.

Theorem 7.3. The moduli spaces of surfaces on the Noether lines are topologically flat over $\operatorname{Spec} \mathbb{Z}$.

PROOF. This follows immediately from the lifting results above.

Remark 7.4. Of course, it would be nicer to prove flatness of these moduli spaces and to understand their scheme structure (singularities, reducedness) in detail. This is equivalent to understanding the deformation theory of our surfaces, e.g., as in [HorQ]. Unfortunately, it seems that the contribution of Du Val singularities on their canonical models to their deformation functors is more complicated than in characteristic zero. We will come back to this in another article.

\section{APPENDix A. DOUble COVERS AND CANONICAL RESOLUTION OF SINGULARITIES}

In this appendix we recall a couple of facts on double covers and canonical resolution of their singularities. These results are somewhat scattered over the literature and divided into several subcases, depending on whether the characteristic is different from 2 or not. In characteristic 2 , the setup is usually divided into the separable and the inseparable case. Here, we provide the material needed to make the arguments of [HorQ, Section 2] and [Hor2, Section 1] work in arbitrary characteristic.

First, a characteristic-free description of double covers is given in [CD, Chapter $0.1]$. In characteristic $\neq 2$, double covers and resolution of their singularities can be described in terms of branch loci, cf. [HorQ, Section 2] and [BHPV, Section III.7]. In characteristic 2, there are two distinct types of double covers: they are either separable in which case we are dealing with Artin-Schreier extensions (of 
simple type), cf. [Ta], or they are purely inseparable and we are dealing with $p$ closed foliations and their singularities, cf. [Hir]. These latter two cases can be uniformly described in the language of $\alpha_{s}$-torsors, see [Ek2, I.(1.10)] or Section 2,

Let $f: X \rightarrow S$ be a generically finite morphism of degree 2 from a normal onto a smooth surface. Let $\nu: X \rightarrow X^{\prime}$ followed by $f^{\prime}: X^{\prime} \rightarrow S$ be the Stein factorization of $f$. Since $X^{\prime}$ is normal and $f^{\prime}$ is finite, it follows that $f^{\prime}$ is flat. Moreover, since $f^{\prime}$ is of degree 2 we obtain a short exact sequence

$$
0 \rightarrow \mathcal{O}_{S} \rightarrow f_{*}^{\prime} \mathcal{O}_{X^{\prime}} \rightarrow \mathcal{L}^{\vee} \rightarrow 0
$$

where $\mathcal{L}^{\vee}$ is an invertible sheaf on $S$. We define $\mathcal{L}$ to be its dual.

Definition A.1. We refer to $\mathcal{L}$ as the invertible sheaf associated with $\pi$.

In characteristic $\neq 2$, the morphism $f^{\prime}$ is Galois and the decomposition of $f_{*}^{\prime} \mathcal{O}_{X^{\prime}}$ into eigensheaves yields a splitting of (9). Moreover, the branch locus of $f^{\prime}$ corresponds to a section of $\mathcal{L}^{\otimes 2}$ and defines the $\mathcal{O}_{S}$-algebra structure on $f_{*}^{\prime} \mathcal{O}_{X^{\prime}}$ uniquely. However, in characteristic 2 , there is no notion of branch locus if $f^{\prime}$ is inseparable. In the separable case this branch locus corresponds to a section of $\mathcal{L}$, which is in general not enough to determine the $\mathcal{O}_{S}$-algebra structure of $f_{*}^{\prime} \mathcal{O}_{X^{\prime}}$.

Since $f^{\prime}$ is flat and its fibers are Artin-algebras of length 2, which are Gorenstein, it follows that $X^{\prime}$ is Gorenstein. More precisely, we have

$$
\omega_{X^{\prime}} \cong f^{\prime *}\left(\omega_{S} \otimes \mathcal{L}\right), \quad \text { hence } \quad K_{X^{\prime}}^{2}=\frac{1}{2}\left(K_{S}+L\right)^{2},
$$

where $\omega_{X^{\prime}}$ denotes the dualizing sheaf of $X^{\prime}$, cf. [CD, Section 0.1]. Since $X^{\prime}$ is Gorenstein, there exists a Cartier divisor on $X^{\prime}$, the canonical cycle $Z_{K}$, which is supported on the exceptional locus of $\nu$ and such that

$$
\omega_{X} \cong \nu^{*} \omega_{X^{\prime}} \otimes \mathcal{O}_{X}\left(-Z_{K}\right) .
$$

If $\nu$ is minimal, i.e., does not contract any $(-1)$-curves, then $Z_{K} \geq 0$. Then, $Z_{K}$ being zero is equivalent to $X^{\prime}$ having only rational singularities, i.e., Du Val singularities in our setup.

We already remarked that $\mathcal{L}^{\otimes 2}$ replaces the notion of a branch divisor. Then we define an invertible sheaf $\mathcal{R}$ by

$$
\omega_{X} \cong f^{*} \omega_{S} \otimes \mathcal{R} \quad \text { such that the familiar formula } \quad \mathcal{O}_{X}\left(Z_{K}\right) \cong f^{*} \mathcal{L} \otimes \mathcal{R}^{\vee}
$$

from [HorQ, Lemma 4] holds true. This $\mathcal{R}$ plays the role of a ramification divisor, although there is in general no distinguished section of this invertible sheaf.

To resolve the singularities of a finite and flat double cover, there is the following procedure, which is classical in characteristic $\neq 2$, cf. [BHPV, Section III.7] and has been shown in characteristic 2 in the separable case in [Ta, Section 2] and in the inseparable case in [Hir, Proposition 2.6].

Proposition A.2 (canonical resolution of singularities). Let $f: X \rightarrow S$ be a finite and flat morphism of degree 2 from a normal onto a smooth surface. Let $x \in X$ be a singular point. Then there exists a sequence of blow-ups in smooth points 
$\pi: \tilde{S} \rightarrow S$ such that the normalized fiber product

$$
\begin{array}{lll}
X & \varpi & \widetilde{X} \\
\downarrow f & & \downarrow \\
S & \stackrel{\pi}{\leftarrow} & \widetilde{S}
\end{array}
$$

yields a resolution of singularities $\varpi: \widetilde{X} \rightarrow X$ and such that $\widetilde{f}$ is again a finite and flat morphism of degree 2.

We need a characteristic-free substitute for [HorQ, Lemma 6]. Thus, we want to determine the effect of a single blow-up and normalized fiber product in the previous proposition, which in general is only a partial desingularization of $X$.

Lemma A.3. In the situation of Proposition A.2 assume that $\pi$ is only a single blow-up in $f(x)$. Let $E$ be the exceptional divisor of $\pi$ and $\widetilde{\mathcal{L}}$ the invertible sheaf associated with $\tilde{f}$. Then there exists an integer $n \geq 0$ such that

$$
\begin{aligned}
\widetilde{\mathcal{L}} & \cong \pi^{*} \mathcal{L} \otimes \mathcal{O}_{\widetilde{S}}(-n E) \\
\omega_{\tilde{X}} & \cong \varpi^{*}\left(\omega_{X}\right) \otimes \widetilde{f}^{*} \mathcal{O}_{\widetilde{S}}((1-n) E) \\
K_{\widetilde{X}}^{2} & =K_{X}^{2}-2(n-1)^{2} \\
\chi\left(\mathcal{O}_{\widetilde{X}}\right) & =\chi\left(\mathcal{O}_{X}\right)-\frac{1}{2} n(n-1) \\
& =2 \chi\left(\mathcal{O}_{S}\right)+\frac{1}{2} \mathcal{L} \cdot\left(\omega_{S} \otimes \mathcal{L}\right)-\frac{1}{2} n(n-1) .
\end{aligned}
$$

Moreover,

(1) $x \in X$ is a smooth point if and only if $n=0$, and

(2) if $x \in X$ is a rational singularity then $n=1$.

Proof. Pulling back (9) to $\widetilde{S}$, we obtain a commutative diagram

$$
\begin{aligned}
& 0 \rightarrow \pi^{*} \mathcal{O}_{S} \rightarrow \pi^{*} f_{*} \mathcal{O}_{X} \rightarrow \pi^{*} \mathcal{L}^{\vee} \rightarrow 0 \\
& 0 \rightarrow \begin{array}{l}
\downarrow \cong \\
\mathcal{O}_{\widetilde{S}}
\end{array} \rightarrow \underset{\widetilde{f}_{*} \mathcal{O}_{\widetilde{X}}}{\downarrow} \rightarrow \stackrel{\downarrow}{\tilde{\mathcal{L}}^{\vee}} \rightarrow 0
\end{aligned}
$$

where $\pi^{*} f_{*} \mathcal{O}_{X} \rightarrow \widetilde{f}_{*} \mathcal{O}_{\widetilde{X}}$ is the normalization. The induced inclusion $\pi^{*} \mathcal{L}^{\vee} \rightarrow \widetilde{\mathcal{L}}^{\vee}$ is an isomorphism outside $E$. Hence there exists an integer $n \geq 0$ such that

$$
\pi^{*} \mathcal{L}^{\vee} \cong \widetilde{\mathcal{L}}^{\vee} \otimes \mathcal{O}_{\widetilde{S}}(-n E)
$$

The formulae for $\omega_{\widetilde{X}}$ and $K_{\widetilde{X}}^{2}$ follow from (10). We compute $\chi\left(\mathcal{O}_{\tilde{X}}\right)$ by taking Euler characteristics in (11) and applying Riemann-Roch.

If $x \in X$ is singular, then $X \times_{S} \widetilde{S}$ is singular along $\varpi^{-1}(x)$. In particular, $\pi^{*} f_{*} \mathcal{O}_{X}$ is not normal and we get $n \geq 1$.

If $x \in X$ has at worst rational singularities then so has $\widetilde{X}$ along $\varpi^{-1}(x)$ and thus $\chi\left(\mathcal{O}_{X}\right)=\chi\left(\mathcal{O}_{\tilde{X}}\right)$, which gives $n \in\{0,1\}$.

Finally, if $x \in X$ is a smooth point, then $\widetilde{X}$ is smooth along $\varpi^{-1}(x)$, which implies that $K_{\widetilde{X}}^{2}<K_{X}^{2}$. In particular, $n \neq 1$ which gives $n=0$ be the previous consideration. 
Remark A.4. In characteristic $\neq 2$, the integer $n$ is half the multiplicity of a singularity of the branch curve, see [HorQ, Lemma 6] or [BHPV], Theorem III.(7.2)].

We note that our singularities are automatically Gorenstein. Thus, rational singularities on double covers are Du Val singularities. The following extends [HorQ, Lemma 5] and [BHPV, Theorem III.(7.2)] to arbitrary characteristic:

Proposition A.5. Let $f: X \rightarrow S$ be a finite and flat morphism of degree 2 , where $S$ is a smooth surface and $X$ has a Du Val singularity in $x \in X$. Then the canonical resolution $\varpi: \widetilde{X} \rightarrow X$ coincides with the minimal resolution of singularities. Moreover,

$$
\widetilde{\mathcal{L}} \cong \pi^{*} \mathcal{L} \otimes \omega_{\widetilde{S}}^{\vee} \otimes \pi^{*} \omega_{S}
$$

Proof. By the previous lemma, we find $n=1$ for every single blow-up in a closed point of the base. Thus, the canonical resolution of singularities fulfills $\omega_{\tilde{X}}=\varpi^{*} \omega_{X}$, which implies that it is minimal.

From the formulae

$$
\omega_{X} \cong f^{*}\left(\omega_{S} \otimes \mathcal{L}\right), \quad \omega_{\widetilde{X}} \cong \widetilde{f}^{*}\left(\omega_{\widetilde{S}} \otimes \widetilde{\mathcal{L}}\right) \quad \text { and } \quad \omega_{\widetilde{X}} \cong \varpi^{*}\left(\omega_{X}\right)
$$

we obtain the statement linking $\pi^{*} \mathcal{L}$ to $\widetilde{\mathcal{L}}$.

\section{REFERENCES}

[Ar] M. Artin, Algebraic Construction of Brieskorn's Resolutions, J. of Algebra 29, 330-348 (1974).

[AM] M. Artin, B. Mazur, Formal groups arising from algebraic varieties, Ann. Sci. École Norm. Sup. 10, 87-131 (1977).

[AK] T. Ashikaga, K. Konno, Algebraic surfaces of general type with $c_{1}^{2}=3 p_{g}-7$, Tohoku Math. J. 42, 517-536 (1990).

[BHPV] W. P. Barth, K. Hulek, C. Peters, A. van de Ven, Compact Complex Surfaces, 2nd edition, Erg. d. Math., 3. Folge Volume 4, Springer (2004).

[Bea] A. Beauville, L'application canonique pour les surfaces de type général, Invent. Math. 55, 121-140 (1979).

[Bo] E. Bombieri, Canonical models of surfaces of general type, Inst. Hautes Études Sci. Publ. Math. 42, 171-220 (1973).

[C] G. Castelnuovo, Osservazioni intorno alia geometria sopra una superficie, Nota II, Rendiconti del R. Instituto Lombardo 24 (1891).

[CD] F.R. Cossec, I.V. Dolgachev, Enriques Surfaces I, Prog. in Math. 76, Birkhäuser (1989).

[DI] P. Deligne, L. Illusie, Relèvements modulo $p^{2}$ et décomposition du complexe de de Rham, Invent. Math. 89, 247-270 (1987).

[EH] D. Eisenbud, J. Harris, On Varieties of Minimal Degree (A Centennial Account), Algebraic Geometry, Bowdoin 1985, Proc. Symp. Pure Math. 46, Part 1, 3-13 (1987).

[Ek1] T. Ekedahl, Diagonal complexes and F-gauge structures, Hermann (1986).

[Ek2] T. Ekedahl, Canonical models of surfaces of general type in positive characteristic, Inst. Hautes Études Sci. Publ. Math. No. 67, 97-144 (1988).

[En] F. Enriques, Le superficie algebriche, Nicola Zanichelli (1949).

[Hart] R. Hartshorne, Algebraic Geometry, Springer (1977).

[Hir] M. Hirokado, Singularities of multiplicative p-closed vector fields and global 1-forms on Zariski surfaces, J. Math. Kyoto Univ., 39-3, 455-468 (1999)

[HorQ] E. Horikawa, On Deformations of Quintic Surfaces, Invent. math. 31, 43-85 (1975).

[Hor1] E. Horikawa, Algebraic surfaces of general type with small $c_{1}^{2}$, I, Ann. Math. 104, 357387 (1976). 
[Hor2] E. Horikawa, Algebraic surfaces of general type with small $c_{1}^{2}$, II, Invent. Math. 37, 121155 (1976).

[Ill] L. Illusie, Complexe de de Rham-Witt et cohomologie cristalline, Ann. Sci. École Norm. Sup. 12, 501-661 (1979).

[Il12] L. Illusie, Grothendieck's existence theorem in formal geometry with a letter of JeanPierre Serre, AMS Math. Surveys Monogr. 123, Fundamental Algebraic Geometry, 179233 (2005).

[Jou] J.-P. Jouanolou, Théorèmes de Bertini et Applications, Prog. in Math. 42, Birkhäuser (1983).

[Kar] U. Karras, Local cohomology along exceptional sets, Math. Ann. 275, 673-682 (1986).

[Ka] N. Katz, Nilpotent connections and the monodromy theorem: applications of a result of Turrittin, Publ. Math. IHES 39, 175-232 (1970).

[Lie1] C. Liedtke, Uniruled surfaces of general type, Math. Z. 259, 775-797 (2008).

[Lie2] C. Liedtke, Algebraic surfaces of general type with small $c_{1}^{2}$ in positive characteristic, Nagoya Math. J. 191, 111-134 (2008).

[Lie3] C. Liedtke, Non-classical Godeaux Surfaces, Math. Ann. 343, 623-637 (2009).

[LS] C. Liedtke, M. Schütt, Unirational Surfaces on the Noether Line, Pacific J. Math. 239, 343-356 (2009).

[Lip] J. Lipman, Rational singularities, with applications to algebraic surfaces and unique factorization, Publ. Math. IHES 36, 195-279 (1969).

[Lip2] J. Lipman, Desingularization of two-dimensional schemes, Ann. Math. 107, 151-207 (1978).

[Mi] R. Miranda, Nonclassical Godeaux surfaces in characteristic five, Proc. Amer. Math. Soc. 91, 9-11 (1984).

[Noe] M. Noether, Zur Theorie der eindeutigen Entsprechungen algebraischer Gebilde, Math. Ann. 8, 495-533 (1875).

[Pa] R. Pardini, Triple covers in positive characteristic, Ark. Mat. 27, 319-341 (1989).

[Ram] C.P. Ramanujam, Remarks on the Kodaira vanishing theorem, Journal of the Indian Math. Soc. 36, 41-51 (1972).

[S-B] N.I. Shepherd-Barron, Unstable vector bundles and linear systems on surfaces in characteristic $p$, Invent. math. 106, 243-262 (1991).

[Ta] Y. Takeda, Artin-Schreier coverings of algebraic surfaces, J. Math. Soc. Japan 41, 415435 (1989).

Department of Mathematics, Stanford University, 450 Serra Mall, Stanford, CA 94305, USA

E-mail address: liedtke@math.stanford.edu 